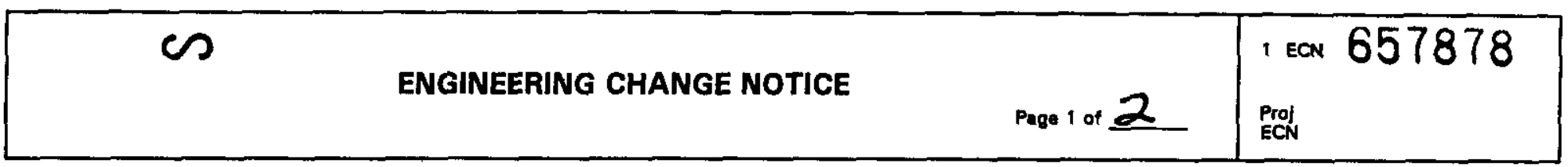

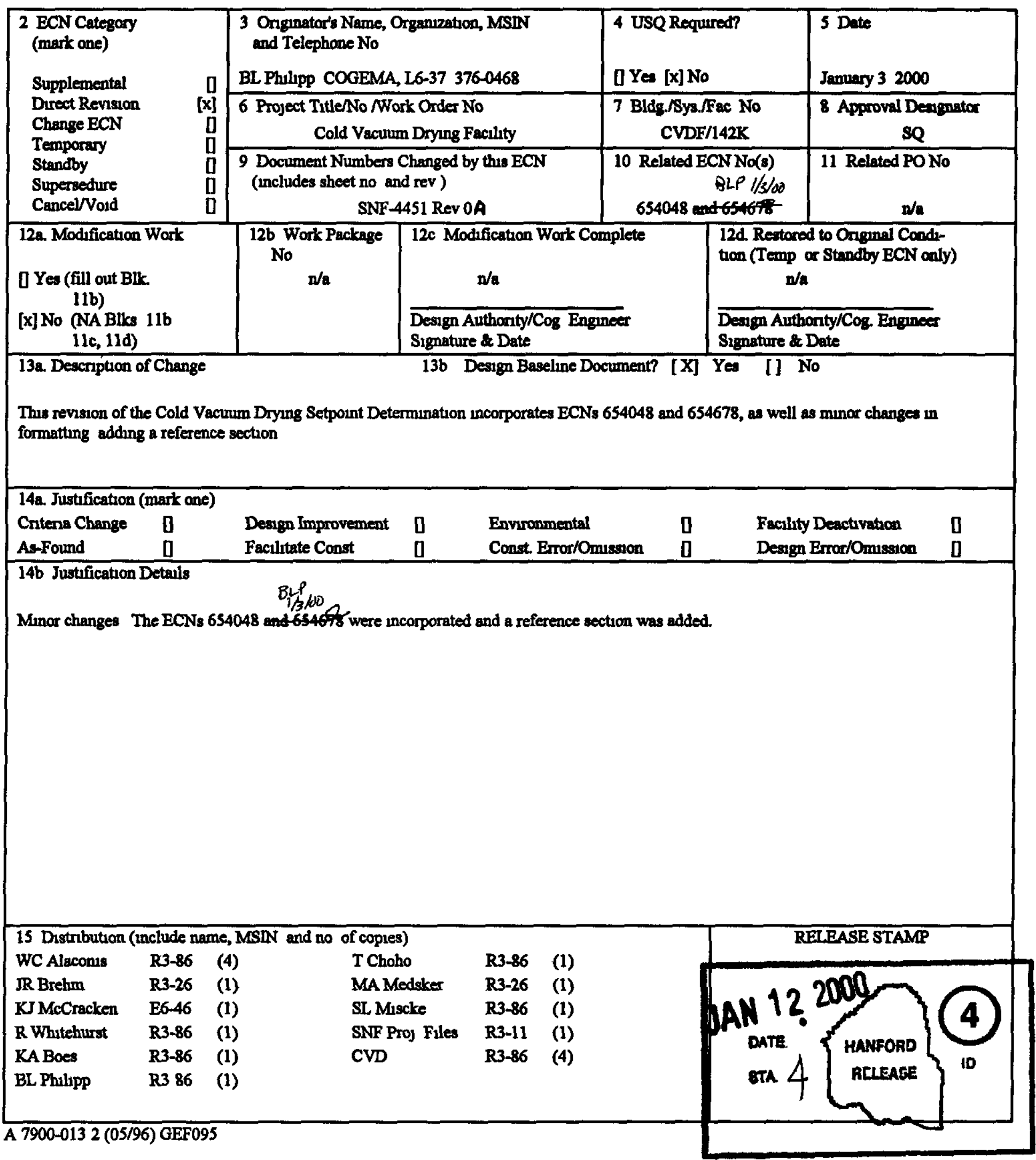




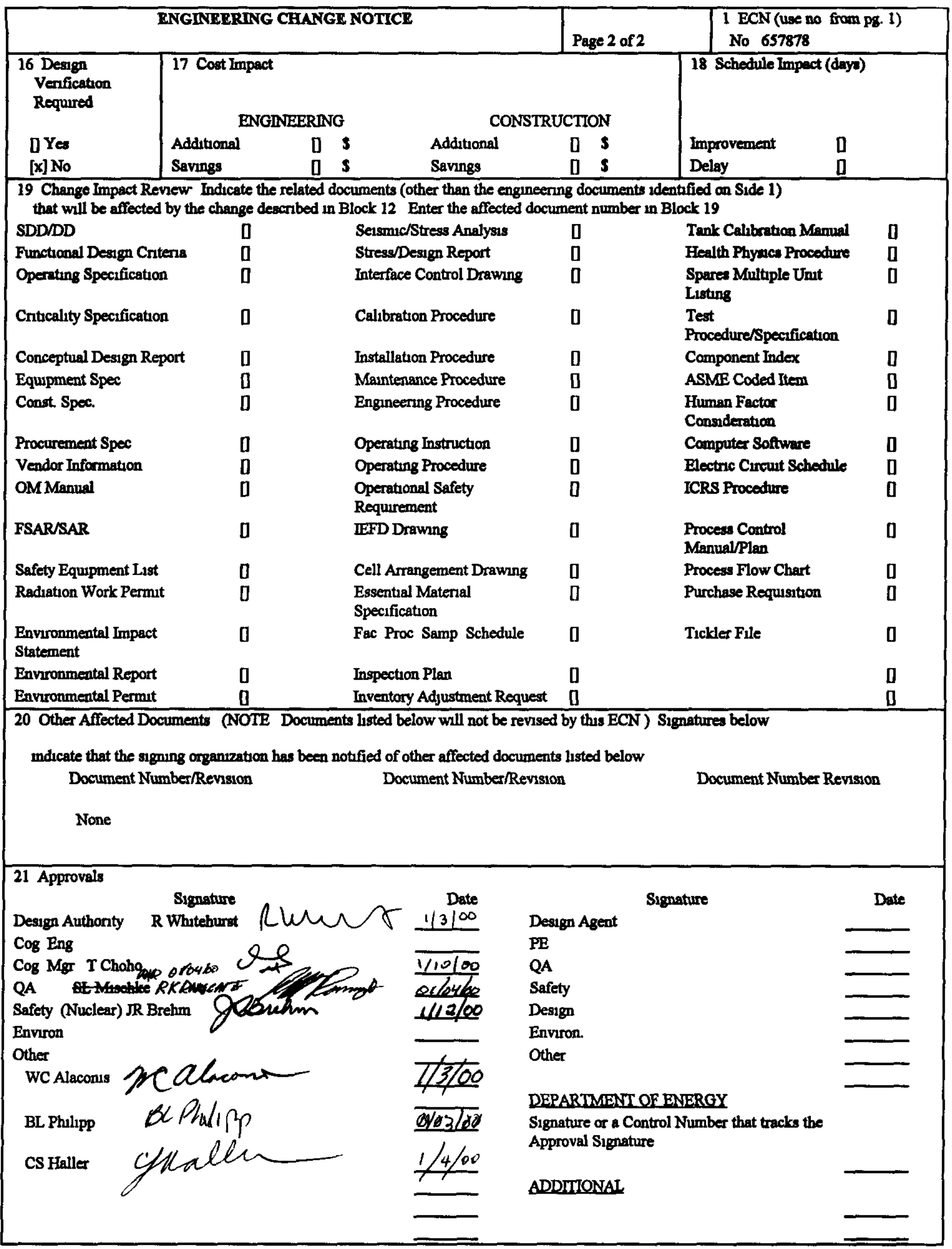




\title{
COLD VACUUM DRYING (CVD) SET POINT DETERMINATION
}

\author{
BL Philipp \\ COGEMA Engineering Corp \\ Richland, WA 09352 \\ US Department of Energy Contract DE-AC06-96RL13200

$\begin{array}{lll}\text { EDT/ECN } & 657878 & \text { UC } \\ \text { Org Code } & & \text { Charge Code } \\ \text { B\&R Code } & 39 \text { Ew7 } 0400 & \text { Total Pages } 52\end{array}$ \\ Key Words CVD, Cold Vacuum Drying, set point, sCIC
}

Abstract This document provides the calculations used to determine the error of safety class signals used for the CVD process These errors are used with the Parameter limits to arive at the initial set point

TRADEMARK DISCLAIMER Rotorence hereln to any speciac commercial product, process or earvice by trade name trademark, manutheturer or otherwtes dose not nocesearly constitute or imply kis endoreement mecommendation or favoring by the United States Government or any agency thoreof or its contractore or subcontractors

Printed in the United States of America To obtain coples of this document contact. Document Control Services P O BOx 950 Mallatop H0-08 Richland WA 90352 Phone (509) 3722420 Fax (509) 376-4989
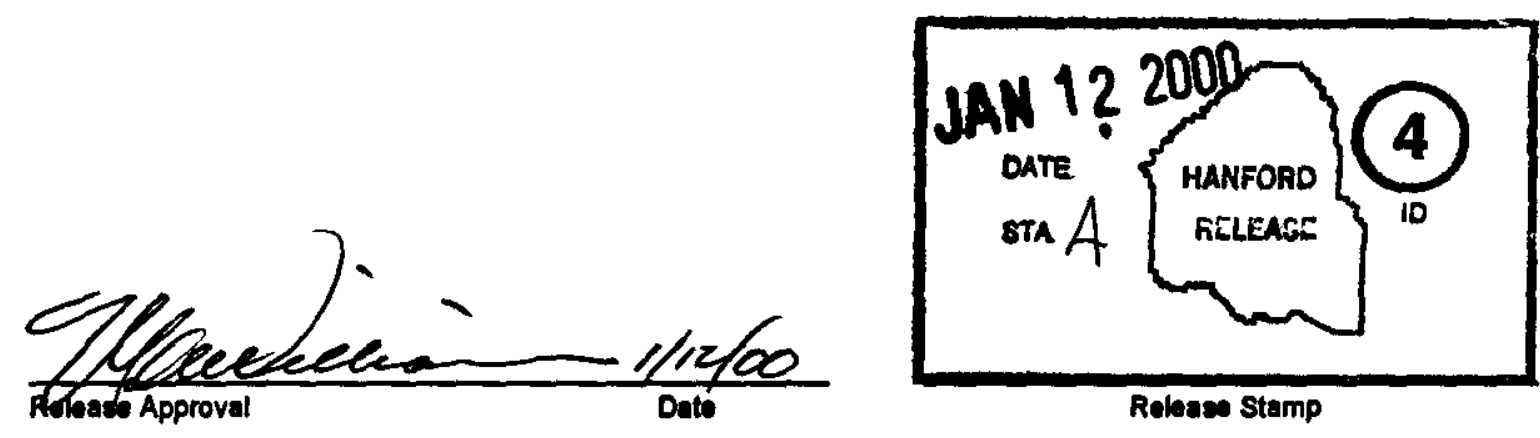

\section{Approved For Public Release}




\begin{tabular}{|c|c|c|c|c|}
\hline \multicolumn{3}{|r|}{ RECORD OI REVIBIOA } & \multicolumn{2}{|l|}{$\begin{array}{l}\text { (1) Document } \\
\text { Number } \\
\text { SNF-4451 }\end{array}$} \\
\hline \multicolumn{5}{|c|}{$\begin{array}{l}\text { (2) Title } \\
\text { COLD VACUUM DRYING SETPOINT DETERMINATION }\end{array}$} \\
\hline \multicolumn{5}{|c|}{ CHANGE CONTROL RECORD } \\
\hline \multirow{2}{*}{$\begin{array}{l}(3) \\
\text { Revialon }\end{array}$} & \multirow{2}{*}{\multicolumn{2}{|c|}{$\begin{array}{l}\text { (4) Description of Change-Replace, Add, } \\
\text { and Delete Pages }\end{array}$}} & \multicolumn{2}{|c|}{ Authorlzed for Release } \\
\hline & & & (5) $\operatorname{cog}$ & (6) Cog Mgr Da \\
\hline 0 & & EDT No 626816 / Original Release & $\begin{array}{l}\text { R Whitehurst } \\
6 / 9 / 99\end{array}$ & $\begin{array}{l}\text { T Choho } \\
6 / 9 / 99\end{array}$ \\
\hline OA & & $\begin{array}{l}\text { ECN No } 654048 \text { Page Replacoment for } \\
\text { changing calibration poriod for ISH- } \\
\text { 1*28, 1*29 from } 1 \text { year to } 1 \text { guarter }\end{array}$ & $\begin{array}{l}\text { R Whitehurst } \\
9 / 9 / 99\end{array}$ & $\begin{array}{l}\text { T Choho } \\
9 / 9 / 99\end{array}$ \\
\hline$R^{2}$ & & $\begin{array}{l}\text { BCN No } 657878 \text { Re-format } \\
\text { Incorporate ECN } 654048 \text { and } 654678\end{array}$ & $\begin{array}{l}\text { R whitehuret } \\
\text { Mut } 1 / 3 / \text { os }\end{array}$ & choho $1 / 10 / \infty$ \\
\hline & & & & \\
\hline & & & & \\
\hline & & & & \\
\hline & & & & \\
\hline & & & & \\
\hline & & & & \\
\hline & & & & \\
\hline & & & & \\
\hline & & & & \\
\hline & & & & \\
\hline & & & & \\
\hline & & & & \\
\hline & & & & \\
\hline & & & & \\
\hline & & & & \\
\hline & & & & \\
\hline & & & & \\
\hline & & & & \\
\hline & & & & \\
\hline & & & & \\
\hline & & & & \\
\hline & & & & \\
\hline & & & & \\
\hline & & & & \\
\hline
\end{tabular}




\section{Cold Vacuum Drying (CVD)}

\section{Set Point Determination}

Project W-441

SNF-4451

January 3, 2000

$\frac{\text { Mubane L Thliph }}{\text { Barbara L Philipp }}$

Electrical I\&C Engineering

COGEMA Engineering Corporation

$\frac{01 / 03 / 2000}{\text { Date }}$

$\frac{1 / 3 / 0^{\circ}}{\text { Date }}$

Tedhnical Reviewer Richard Whitehurst II

Technical Integration

Fluor Daniel Hanford, Incorporated 
Contents

1 INTRODUCTION 6

11 GENERAL $\quad 6$

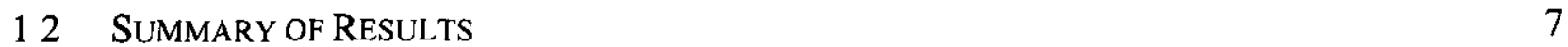

2 MCO VACUUM PRESSURE TRANSMITTER TO SCIC 8

21 VACUUM SET POINT IDENTIFICATION

211 Function $\quad 8$

22 Plant Operating Conditions $\quad 8$

221 Destgn-basts event conditions 9

23 Absolute Pressure TransmitTer (PT-1*08, PT-1*10) 10

231 Instrument Data 10

232 Reference Specifications $\quad 10$

233 Project Specifications $\quad 10$

234 Uncertainty Terms that Affect Set Point Determination 11

24 FRAMATOME STAR SYSTEM 13

241 Processor Module 13

242 Project Specifications $\quad 13$

243 Uncertainty Terms that Affect Set Point Determination 14

25 TOtal LoOP UNCERTAINTY - 12 TORR TRIP SIGNAL LOOP (SC) 15

26 Set Point Determination $\quad 15$

27 Delta Pressure ACCURACY 16

271 Uncertainty Terms that Affect Accuracy 16

3 MCO PRESSURE TRANSMITTER TO SCIC $\quad 18$

31 Set Point IDENTIFICATION $\quad 18$

311 Vacuum Trip $\quad 18$

312 MCO Low Pressure Trip $\quad 18$

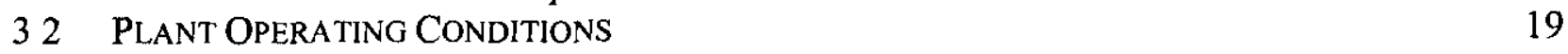

321 Vacuum Trip - Accidents and responses 19

322 Low Pressure Trip - Accldents and responses 19

33 Gauge Pressure Transmitter (PT 1*36, PT-1*37) 19

331 Instrument Data 19

332 Reference Spectfications

333 Project Specifications 19

334 Calibration $\quad 20$

34 FRAMATOME STAR SYSTEM $\quad 22$

341 Processor Module $\quad 22$

342 Project Specifications $\quad 22$

343 Uncertainty terms that affect Callbration 22

35 TOTAL LOOP UNCERTAINTY - SAFETY CLASS LOOP 24

36 SET POINT DETERMINATION 24 
4 TEMPERATURE SWITCH / TRANSMITTER TO SCIC

41 SET POINT IDENTIFICATION 25

411 TW Annulus Water Temperature 25

412 High Bay Temperature 25

42 Plant OPERATING CONDITIONS 25

421 TW Annulus Water Temperature 25

422 High Bay Temperature 25

43 Temperature SWITCH / TRANSMITTER (TSH-1*28, $1 * 29$ TSHH-1*38 1*39) 26

431 Instrument Data 26

432 Reference Specifications 26

433 Project Spectfications $\quad 26$

434 Calibration 26

44 SET Point Determination 29

5 MINIMUM PURGE FLOW TRANSMITTER TO SCIC 30

51 SET POINT IDENTIFICATION 30

511 PWC Low Purge Flow Alarm 30

52 MASS Flow TRANSMITTER (FIT $1 * 20$, FIT- $1 * 21$ ) 30

521 Instrument Data 30

522 Reference Specifications $\quad 30$

523 Project Specifications 31

524 Calibration 31

53 FRAMATOME STAR SYSTEM 33

531 Processor Module 33

532 Project Spectfications

533 Calibration 33

54 TOTAL LOOP UNCERTAINTY - SAFETY Class TRIP SIGNAL LOOP 35

55 SET POINT DETERMINATION 35

6 SEISMIC TRANSMITTER TO SCIC 36

61 Seismic Set Point IDENTIFICATION 36

611 Function 36

62 Plant OPERATING Conditions

621 Design-basis event conditions

63 SEISMic TRANSMITTER (ATR-5335, ATR-5436, ATR-5537) 38

631 Servo Accelerometer 38

632 Strong Motion Recorder 38

633 Project Spectfications $\quad 39$

634 Uncertainty Terms that Affect Set Point Determination 39

64 Set Point Determination $\quad 40$

7 DIFFERENTIAL PRESSURE SWITCH

71 PREsSURE TRANSMITTER (PDIS-8022,8042,8043) 42

711 Instrument Data $\quad 42$

712 Reference Specifications $\quad 42$

713 Project Specificatıons 42 
714 Calibration

8 HE BOTTLE PRESSURE INDICATOR $\quad 45$

81 Gauge Pressure Indicator (PI-5*02 PT 5*21, PT-5*41, PT-5*61) 45

811 Instrument Data 45

812 Reference Speclfications 45

813 Project Specifications $\quad 45$

814 Calibration 45

9 FLOW SWITCH $\quad 48$

91 FLoW SWITCH (FS-8*52)

911 Instrument Data $\quad 48$

912 Reference Specifications 448

913 Caltbration 48

10 REFERENCES $\quad 50$

101 VENDER INFORMATION

102 HANFORD $\quad 50$

\section{List of Figures}

Figure 21 Absolute Pressure Simplified Block Diagram 8

Figure 31 Rosemount GaUge Pressure SiMPlified Block Diagram 18

Figure 51 FCI MaSS Flow TransmitTer Simplified Block Diagram 30

Figure 61 SEISMiC SENSOR Simplified Block Diagram 36

Figure 71 DWyer Differential Pressure Switch Simplified Block Diagram 42

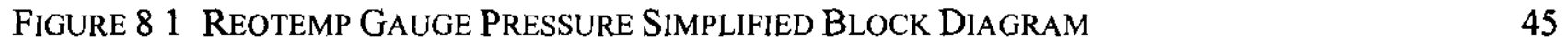

Figure 91 W E ANDERSON (DWyer) VANe OPERATEd Flow SWITCH Simplified Block DIAGRAM

List of Tables

TABle 1-1 SCIC TRIP SETPOINTS, InSTRUMENT ERROR, AND DElay Times (2 SheETS) 


\section{List of Acronyms and Abbreviations}

\begin{tabular}{ll} 
AL & Analytical Limit \\
AU & Accuracy Uncertainty \\
AV & Allowable Value \\
AVSTM & Allowable Value Setpoint Trip Margin \\
$\beta$ & Truncation error \\
B & Bias \\
CS & Calibrated Span \\
CU & Channel Uncertainty \\
DAC & Data Acquisition \\
DR & Drift Effect \\
HE & Humidity Effect \\
FS & Full Scale \\
MCS & Monitoring and Control System \\
mR & milli-Rad \\
MTE & Measurement and Test Equipment \\
NPH & National Phenomena Hazard \\
PC & Performance Category \\
Psig & Pounds per Square Inch - Gauge \\
PSE & Power Supply Effect \\
RA & Relative Accuracy \\
RD & Reading Error \\
Rdg & Reading \\
RE & Radiation Effect \\
RH & Relatıve Humidity \\
SA & Setting Accuracy \\
SC & Safety Class \\
SCC & Standard cubic centimeters \\
SCIC & Safety Class Instrumentation and Controls \\
SE & Seismic Effect \\
SMTE & Sensor Measurement and Test Equipment Effect \\
SP & Static Pressure Effect \\
TDR & Turn Down Ratio \\
TE & Temperature Effect \\
TID & Total Integrated Dose \\
TS & Trip Setpoint \\
URL & Upper Range Limit \\
VDC & Volts - Direct Current \\
\hline
\end{tabular}




\section{Introduction}

\section{General}

The Safety Class Instrumentation and Control (SCIC) system provides active detection and response to process anomalies that, if unmitigated would result in a safety event Specifically actuation of the SCIC system includes two portions The portion which isolates the MCO and initiates the safety-class helium ( $\mathrm{SCHe}$ ) purge, and the portion which detects and stops excessive heat input to the MCO on high tempered water MCO inlet temperature For the MCO isolation and purge the SCIC receives signals from MCO pressure (both positive pressure and vacuum) helium flow rate, bay high temperature switches, seismic trips and tıme under vacuum trips

The SCIC system wll isolate the MCO and start a SCHe system purge if any of the following occur

A Manually initiated 1solation and purge from one of the SCHe 'isolation" and "purge" buttons (administratively controlled)

B Exceeding 8 hours at vacuum the first vacuum cycle or 4 hours at vacuum all subsequent vacuum cycles without re pressurnzing the MCO for a minimum of 4 hours This is referred to as the $8 / 4 / 4$ requirement and provides thermal equilibrium within the MCO

C MCO is below atmospheric pressure and the helium flow is below the minimum required to keep hydrogen less than $4 \%$ by volume When MCO pressure is below 12 torr there is insufficient hydrogen to exceed the $4 \%$ level and therefore no purge is required A five minute time delay on low flow allows flow to be stopped in order to reach $<12$ torr

D During the transition from above atmosphere to vacuum time to reach less than $-117 \mathrm{psig}$ (155 torr) shall not exceed 5 minutes

E During the transition from below $111 \mathrm{psig}(\sim 185$ torr $)$ back to greater than 05 psig pressure the time shall not exceed 5 minutes

F MCO reaches an incorrect pressure state without adequate, verified purge volume The MCO must be maintained above a positive pressure (approximately $05 \mathrm{psig}$ ) to prevent oxygen ingress unless a purge of adequate volume has been completed During bulk water draining the MCO must remain above atmospheric pressure

G Bay temperature exceeds the high temperature trip point of $105^{\circ} \mathrm{F}$ which would impact safety class instrument calibration

H Seismic event of sufficient magnitude (below the Unıform Bulling Code levels)

Set point determination is required for trips $\mathrm{C}$ through $\mathrm{H}$

Set points determination for additional safety sıgnificant sensors used by the HVAC system or the SCHe system are also required 
CVD Set point Determination

\section{Summary of Results}

Table 1-1 Instrument Error

\begin{tabular}{|c|c|c|c|c|}
\hline $\begin{array}{c}\text { Safety Class or } \\
\text { Safety Significant } \\
\text { trip or alarm }\end{array}$ & Field sensor & $\begin{array}{c}\text { Total instrument } \\
\text { error }\end{array}$ & Parameter limit & SCIC trip setpoint \\
\hline $\begin{array}{l}\text { MCO } 12 \text { torr } \\
\text { purge bypass }\end{array}$ & PT $1 * 08,1 * 10$ & $\begin{array}{l}2 \text { torr with } 2 \text { torr } \\
\text { system drop }\end{array}$ & $\leq 12$ torr & $\leq 80$ torr \\
\hline $\begin{array}{l}\text { MCO high } \\
\text { pressure }\end{array}$ & PT $1 * 36,1 * 37$ & $026 \mathrm{ps} 1 \mathrm{~g}$ & $\leq 25$ psig & $\leq 12 \mathrm{psig}$ \\
\hline $\begin{array}{l}\text { MCO low } \\
\text { pressure }\end{array}$ & PT $1 * 36,1 * 37$ & $026 \mathrm{psig}$ & $\geq 024 \mathrm{psig}$ & $\geq 05$ psig \\
\hline $\begin{array}{l}\text { Vacuum limit } \\
\text { timer }\end{array}$ & PT $1 * 36,1 * 37$ & $026 \mathrm{psig}$ & $\geq 0 \mathrm{psig}$ & $\geq 026 \mathrm{psig}$ \\
\hline $\begin{array}{l}\text { MCO pressure } \\
\text { decay fall }\end{array}$ & PT $1 * 36,1 * 37$ & $\begin{array}{c}026 \mathrm{psig} \text { and } \\
03 \mathrm{psig}\end{array}$ & $\begin{array}{l}>024 \mathrm{psig} \text { and } \\
\leq-114 \mathrm{psig}\end{array}$ & $\begin{array}{l}\geq 05 \mathrm{psig} \text { and } \\
\leq-117 \mathrm{psig}\end{array}$ \\
\hline $\begin{array}{l}\text { MCO pressure } \\
\text { rise fail }\end{array}$ & PT 1*36 1*37 & $\begin{array}{c}03 \text { psig and } \\
026 \mathrm{psig}\end{array}$ & $\begin{array}{l}\leq-108 \text { psig and } \\
\geq 024 \text { psig }\end{array}$ & $\begin{array}{c}\leq 111 \mathrm{psig} \text { and } \\
\geq 05 \mathrm{psig}\end{array}$ \\
\hline $\begin{array}{l}\text { TW annulus } \\
\text { water temperature }\end{array}$ & TSH $1 * 28,1 * 29$ & $19^{\circ} \mathrm{C}$ & $\leq 50^{\circ} \mathrm{C}$ & $\leq 481{ }^{\circ} \mathrm{C}$ \\
\hline $\begin{array}{l}\text { High bay } \\
\text { temperature }\end{array}$ & TSHH $1 * 38,1 * 39$ & $56^{\circ} \mathrm{F}$ & $\leq 95^{\circ} \mathrm{F}$ & $\leq 89^{\circ} \mathrm{F}$ \\
\hline $\mathrm{MCO}$ pre-purge & FIT $1 * 20,1 * 21$ & $04 \mathrm{scfm}$ & $280 \mathrm{scfm}$ & $284 \mathrm{scfm}$ \\
\hline $\begin{array}{l}\text { MCO low purge } \\
\text { flow }\end{array}$ & FIT $1 * 201 * 21$ & $04 \mathrm{scfm}$ & $\geq 07 \mathrm{scfm}$ & $211 \mathrm{scfm}$ \\
\hline Selsmic & $\begin{array}{c}\text { ATR } 52355336 \\
5437\end{array}$ & $001 \mathrm{~g}$ & $\leq 006 \mathrm{~g}$ & $\leq 005 \mathrm{~g}$ \\
\hline $\begin{array}{l}\text { HVAC delta- } \\
\text { pressure switch }\end{array}$ & $\begin{array}{c}\text { PDIS } 80228042, \\
8043\end{array}$ & $0013 \mathrm{in} \mathrm{H}_{2} \mathrm{O}$ & & \\
\hline $\begin{array}{l}\text { SCHe Bottle } \\
\text { Pressure }\end{array}$ & $\begin{array}{c}\mathrm{PI}-5 * 02,5^{*} 21 \\
5 * 41,5^{*} 61\end{array}$ & $56 \mathrm{psig}$ & & \\
\hline $\begin{array}{l}\text { HVAC Flow } \\
\text { switch }\end{array}$ & FS-8*52 & $120 \mathrm{scfm}$ & & \\
\hline
\end{tabular}




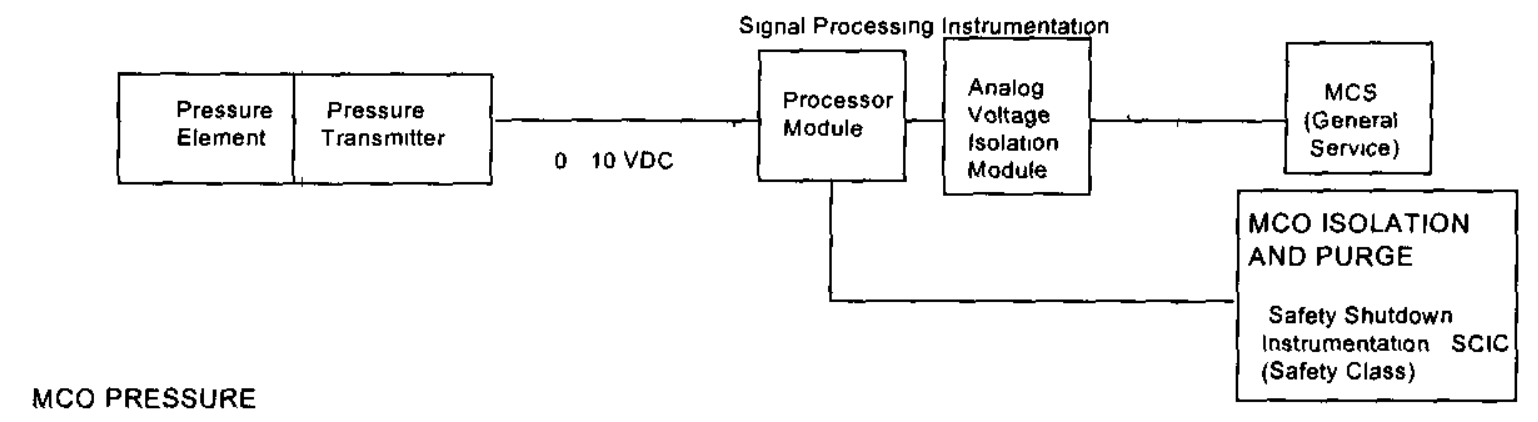

\section{$2 \mathrm{MCO}$ vacuum pressure transmitter to SCIC}

Figure 21 Absolute Pressure Simplified Block Diagram

\section{Vacuum Set Point Identification}

Durng the Drying mode the SCIC system will isolate the MCO and start a SCHe system purge if the $\mathrm{MCO}$ is below atmospheric pressure and the helium flow is below the minimum required to keep hydrogen less than $4 \%$ by volume When MCO pressure is below 12 torr there is insufficient hydrogen to reach flammable conditions upon an air ingress and therefore no purge is required The 12 torr limit is the analytical limit for the vacuum pressure monitors PT $1 * 08, \mathrm{PT}$ $1 * 10$

\section{Function}

A five minute time delay on low flow allows flow to be stopped in order to reach $<12$ torr Once pressure increases above the 12 torr parameter limit, there is an 2 minute delay to allow a re start of flow A trip will occur after this 2 minutes if flow is not above the minimum required or pressure is not less than 12 torr

\section{Plant Operating Conditıons}

Durnng the Drying Mode, the Safety Class Instrumentation and Control System (SCIC) has a MCO Vacuum Timer (initialized to 8 hours) that is used to venfy that the Monitoring and Control System (MCS) operates each of the vacuum drying steps within the correct time frame The MCS operates a continuous purge of helium (at a rate of approximately $15 \mathrm{scfm}$ ) through the MCO during the initial vacuum Drying mode The helium purge of $15 \mathrm{scfm}$ is maintained to assure that hydrogen does not accumulate This flow rate establishes a concentration inside the MCO which would be less than $\sim 4 \%$ (not including sensor error) for boundary fuel This ensures that the minimum required TSR of $11 \mathrm{scfm}$ (parameter limit plus setpoint error) is not violated This mode of operation is continued until the MCO is dried to a point that allows a pressure reduction to below 12 torr for a maximum time period of 8 hours 
Ideally, the system will remain below 12 torr for the entire 8 hours However, if pressure can not be reduced to less than 12 torr within the 8 hour period, the operator can use the MCS to proceed to the thermal reset During the thermal reset the MCS isolates the vacuum pump and inttiates a backfill of the MCO with helium It increases the MCO pressure to $1 \mathrm{psig}$, and maintains a helium purge of approximately $15 \mathrm{scfm}$ for 4 hours to reduce thermal gradients within the fuel The four hour thermal re set is a safety requirement

Following the helium backfill and purge for 4 hours, the MCO vacuum timer is re-set to 4 hours and a second evacuation of the MCO is performed The maximum time for the second and any subsequent evacuations is 4 hours Each subsequent evacuation is followed by a 4-hour minımum duration of helium backfill and purge

Once the condenser is no longer removing water the condenser is isolated, the flow is routed through a condenser bypass line and the vacuum pump operates until pressure equalizes based on the minimum helium flow injection A short duration is allowed to secure the purge and reach a pressure below 12 torr Once 12 torr is reached, operation without helium purge is allowed and pressure is expected to be below 05 torr The MCO pressure is monitored and upon reaching a pressure of $<05$ torr, the MCO is isolated At this point, a dryness verification test is performed by means of a pressure rebound test

If the pressure rises above 3 torr withın an hour (pressure rebound test), insufficient drying has taken place and more drying is required More vacuum pumping is performed if the test fatls Once the MCO passes the pressure rebound test, an evacuation to less than 30 torr for up to 28 hours is then conducted with the expected system base pressure of 01 torr is achieved This is called the "PROOF" mode of operation The proof mode requires the use of the vacuum pump only, while a residual gas analyzer samples the buld-up of impurities within the MCO atmosphere during this operation Multiple faulted conditions could result in water addition to the $\mathrm{MCO}$ therefore a second and final rebound test is conducted at the end of the proof mode This post drying operation (1 e "PROOF" mode or mode 6) at $46^{\circ} \mathrm{C}$ is utılized to verify that $200 \mathrm{~g}(044 \mathrm{lb})$ of water or less is in the MCO prior to shipment to the CSB (see HNF-1851 Cold Vacuum Drying Restdual Free Water Test Description)

\section{Design-basis event conditions}

1 The SCIC will initiate a trip during Drying mode if the MCO pressure exceeds the set point for the 12 torr parameter limit during the 8 hour vacuum interval or the 4 hour vacuum intervals for more than 2 minutes

2 The SCIC will initiate a trip during Proof mode if the MCO pressure exceeds the set point for the 12 torr parameter limit 


\section{Absolute Pressure Transmitter (PT-1*08, PT-1*10)}

The sensor chosen to monitor pressure for the 12 torr analytical limit is a high accuracy, temperature regulated Baratron ${ }^{\text {tM }}$ Absolute Pressure Transmitters from MKS Instruments This device is designed for industrial vacuum applications, and comes standard with Factory Mutual Approvals for a NEMA 4 housing

\section{Instrument Data}

Name Baratron Absolute Pressure Transmitter

Manufacturer MKS Instruments Inc

Model Number 427A A 00100

Type High Accuracy, Temperature Controlled $\left(45^{\circ} \mathrm{C}\right)$ Absolute Pressure Transmitter

232 Reference Specifications (Bulletın 400-10/96, 1996 - MKS Instruments, Inc )

Range 0 to 100 Torr

Resolution $1 \times 10^{4}$ of Full Scale

Accuracy $015 \%$ of Reading \pm temperature coefficient

Temperature Effect Zero $0007 \% \mathrm{FS} /{ }^{\circ} \mathrm{C}$

Span $0020 \% \operatorname{Rdg} /{ }^{\circ} \mathrm{C}$

Temperature Limits $15-40^{\circ} \mathrm{C}\left(60-105^{\circ} \mathrm{F}\right)$

Uncertainty below range $15^{\circ} \mathrm{C}\left(60^{\circ} \mathrm{F}\right)= \pm 005$ torr

(letter from R Traverso MKS Applications Engıneer 1/26/98)

Power Supply \pm 15 VDC $\pm 5 \%$

Input/Output Range 00 to $100 \mathrm{VDC}$

Sensor Overpressure limit 35 psia

Factory Mutual Approvals Explosion-proof Class 1 Division 1\&2, Group C \& D

Calibration Interval 1 year between calibrations

\section{Project Specifications}

The project safety specifications for the 12 torr sensor include the following

Safety Classification Safety Class (SC)

Performance Category PC-3 (for pressure boundary only)

Environmental Qualification Environmental Condition B (Certıfied to $105^{\circ} \mathrm{F}$ )

NPH Design Requirements Seismic Condition B for boundary only

Required Safety Functions Pressure boundary integnity, input to SCIC for 12 torr trip and pressure rebound test results

\footnotetext{
"Baratron is a registered trademark of MKS Instruments, Inc, Andover MA
} 
MCO Vacuum transmitter

234 Uncertainty Terms that Affect Set Point Determination

A Assumptions

1) Turn Down Ratio $=1$ (transmitter will have a full span of 100 Torr)

11) Calibrations performed at ambient $72 \pm 8^{\circ} \mathrm{F}$ or $64-80^{\circ} \mathrm{F}$

111 ) $\mathrm{Min} / \max$ temperature range $1 \mathrm{~s} 40-115^{\circ} \mathrm{F}$

iv ) Drift $\pm 1 \%$ Calıbrated Span (CS) per year

v) Calıbrated Span (CS) $=100$ Torr

B Sensor Uncertainty

1) Relative Accuracy (RA)

Given RA $=0$ 15\% Reading (Rdg)

11) Static Pressure (SP)

$\mathrm{SP}=0 \% \mathrm{CS}$

Basis Assumed zero for pressures $<35$ psı

Sensor maintains RA $= \pm 015 \%$ of Rdg or $120 \%$ Full Scale whichever is

larger up to $35 \mathrm{ps} 1$

111 ) Humidity Effect (HE)

$\mathrm{HE}=0 \%$ Calıbrated Span

Basis Housed in NEMA 4 enclosure

1V ) Temperature Effect (TE)

Given $\mathrm{TE}_{\mathrm{s}}= \pm 0020 \% \mathrm{Rdg} /{ }^{\circ} \mathrm{C}$ for Span (100 Torr)

$\mathrm{TE}_{\mathrm{z}}= \pm 0007 \% \mathrm{FS} /{ }^{\circ} \mathrm{C}$ for Zero (0 Torr)

Uncertainty below temp range of $15^{\circ} \mathrm{C}\left(60^{\circ} \mathrm{F}\right)= \pm 005$ torr

With $\mathrm{TDR}=1 \mathrm{CS}=\mathrm{Span}=100$ Torr

Assume worst case $\mathrm{Rdg}=100 \quad \mathrm{TE}_{\mathrm{s}}=002 \% \mathrm{CS} /{ }^{\circ} \mathrm{C}$

$\mathrm{TE}_{\mathrm{z}}=0007 \% \mathrm{CS} /{ }^{\circ} \mathrm{C}$

Combining terms (+)

Temp Effect $=0007 \% * \mathrm{FS} /{ }^{\circ} \mathrm{C}+002 \% * \mathrm{Rdg} /{ }^{\circ} \mathrm{C}=0027 \% \mathrm{CS} /{ }^{\circ} \mathrm{C}$

Temp Uncertainty $=0027 \% \mathrm{CS} /{ }^{\circ} \mathrm{C}+005 * 100 \% / 100$ torr (converts to $\mathrm{CS}$ since calibrated range is 100 torr) $=0027 \% \mathrm{CS} /{ }^{\circ} \mathrm{C}+005 \% \mathrm{CS}$

Assume $T \max =105^{\circ} \mathrm{F}$

Tnorm (lower) $=72-8=64^{\circ} \mathrm{F}$

Therefore Tdelta $=41 \Delta^{\circ} \mathrm{F}=228 \Delta^{\circ} \mathrm{C}$ 
MCO Vacuum transmitter

$$
\begin{aligned}
& \text { 1 e }, \text { Temp Uncertainty }=\left(0007^{*} \mathrm{FS} * \mathrm{dT}+002 * \mathrm{Rdg} * \mathrm{dT}+005^{*} \mathrm{FS}\right) \%= \\
& \left(0007^{*} 100 * 228^{\circ} \mathrm{C}+002^{*} 100 \text { torr }^{*} 228^{\circ} \mathrm{C}+005^{*} 100\right) / 100 \\
& =0666 \% \mathrm{CS}
\end{aligned}
$$

v) Radiation Effect (RE)

Given Dose Rate $=85 \mathrm{mR} / \mathrm{hr}$

Total Integrated Dose $=85 \mathrm{mR} / \mathrm{hr} * 43800 \mathrm{hr}=372,300 \mathrm{mR}$

No Radiation Effect Data assumed zero because relatıvely small dose

Assume $\mathrm{RE}= \pm 0 \% \mathrm{CS}$

v1 ) Power Supply Effect (PSE)

Given No PSE while supply voltage maintained at 15 VDC $\pm 5 \%$

$\mathrm{PSE}=0 \%$

vi1 ) Seismic Effect (SE)

No credit for this device is taken during or after a seismic event

$\mathrm{SE}=0 \%$

v111 ) Sensor Measurement and Test Equipment Effect (SMTE)

Test Equipment 1 -Fluke 45, DC voltage mode with a 5 digit display

Relative accuracy - measurement and test equipment

$$
\begin{aligned}
\mathrm{RA}_{\mathrm{MTE} 1} & =0025 \% \mathrm{Rdg}+2 \mathrm{~d} \text { gits } \\
& =0025 \% *(100 \mathrm{torr} \operatorname{Rdg} /(100 \mathrm{torr} / \mathrm{CS}))+0002 \mathrm{~V} /(10 \mathrm{~V} / \mathrm{CS}) * 100 \\
& = \pm 0045 \% \mathrm{CS}
\end{aligned}
$$

Relative accuracy - calıbration standard

$\mathrm{RA}_{\mathrm{STD} 1}=0 \% \mathrm{CS}$

$\mathrm{RD} 1=0 \% \mathrm{CS}$

$\mathrm{SAl}=(0025 / 10)^{*} 100=025 \% \mathrm{CS}($ accuracy/upper range lımit $(10 \mathrm{VDC}) * \mathrm{CS}$ of instrument (100 torr)

$\mathrm{MTE} 1=\left(\mathrm{RA}_{\mathrm{MTE}}{ }^{2}+\mathrm{RA}_{\mathrm{STD1}}{ }^{2}+\mathrm{RD}^{2}+\mathrm{SA} 1^{2}\right)^{1 / 2}$

$\mathrm{MTE} 1=\left(0045^{2}+0^{2}+0^{2}+025^{2}\right)^{\prime}=0254 \% \mathrm{CS}$

Test Equipment2, Heise PTE-1 with HQS-2 sensor (0-100 Torr)

$\mathrm{RA}_{\mathrm{MTE} 2}= \pm 01 \% \mathrm{CS}$

$\mathrm{RA}_{\mathrm{STD} 2}= \pm 001 \% \mathrm{CS}$

$\mathrm{RD} 2=0 \% \mathrm{CS}$

$\mathrm{SA} 2=0 \% \mathrm{CS}$

$\mathrm{MTE} 2=\left(\mathrm{RA}_{\mathrm{MTE} 2}{ }^{2}+\mathrm{RA}_{\mathrm{STD} 2}{ }^{2}+\mathrm{RD}^{2}+\mathrm{SA}^{2}\right)^{1 / 2}$

$\mathrm{MTE} 2=\left(01^{2}+001^{2}+0^{2}+0^{2}\right)^{\prime}=01005 \% \mathrm{CS}$

$\mathrm{SMTE}=\mathrm{MTE} 1+\mathrm{MTE} 2=0254+01005=0355 \% \mathrm{CS}$ 
1x ) Total Sensor Uncertainties

The assumed drift term DR will be taken as a bias (conservatıve option)

$$
\begin{aligned}
\mathrm{e}^{+} & =\left[(\mathrm{RA}+\mathrm{TE})^{2}+\mathrm{SP}^{2}+\mathrm{HE}^{2}+\mathrm{RE}^{2}+\mathrm{PSE}^{2}+\mathrm{SE}^{2}+\mathrm{SMTE}^{2}\right]^{\prime}+\mathrm{DR} \\
& =\left[(015+0666)^{2}+0+0+0+0+0+(355)^{2}\right]^{1 / 2}+1=19 \% \mathrm{CS} \\
\mathrm{e} & =-\left(\left[(\mathrm{RA}+\mathrm{TE})^{2}+\mathrm{SP}^{2}+\mathrm{HE}^{2}+\mathrm{RE}^{2}+\mathrm{PSE}^{2}+\mathrm{SE}^{2}+\mathrm{SMTE}^{2}\right]^{\prime}+\mathrm{DR}\right) \\
& =-\left(\left[(015+0666)^{2}+0+0+0+0+0+(355)^{2}\right]^{1 / 2}+1\right)=19 \% \mathrm{CS}
\end{aligned}
$$

The error associated with the sensor $\left(\mathrm{e}_{\text {sens }}\right)$ is as follows

$$
\mathrm{e}_{\mathrm{sens}}= \pm 19 \% \mathrm{CS}
$$

\section{Framatome STAR System}

\section{Processor Module}

2411 Instrument Data

Name Processor Module

Manufacturer Framatome Technologies

Model Number 1225275-002

Type Signal Processing Module

2412 Reference Specıficatıons (1960501R 5/96 - Framatome Technologies)

Range 0 to $10 \mathrm{VDC}$

Resolution 14 bits (DAC)

Drift $\pm 2 \mathrm{mVDC}= \pm 002 \%$ Full Scale

Accuracy $\pm 014 \%$ of Full Range (Analog Input)

$\pm 01 \%$ of Full Range (Analog Output)

Analog Input/Output Range $00 \mathrm{VDC}$ to $100 \mathrm{VDC}$

Calibration Interval 6 months between calibrations

\section{Project Specifications}

Safety Classification Safety Class (SC)

Performance Category PC-3

Environmental Qualification Environmental Condition B

NPH Design Requirements Seismic Condition C

Required Safety Functions H2 Explosion prevention, Runaway Reaction Prevention 
MCO Vacuum transmitter

243 Uncertainty Terms that Affect Set Point Determination

A Assumptions

1) Turn Down Ratio $=1$

11) Calibrations performed at ambient $72 \pm 8^{\circ} \mathrm{F}$ or $64-80^{\circ} \mathrm{F}$

111 ) $\mathrm{Min} / \max$ design temperature is $40-115^{\circ} \mathrm{F}$

iv ) Calibrated Span (CS) $=$ Full Scale $=10 \mathrm{VDC}$

v ) Processor Module input error calculation multiplier factor $=1$

B Channel Uncertainty Safety Class Trip Signal

1) Drift (DR)

$\mathrm{DR} 1=002$

$\mathrm{DR}= \pm 002 \% \mathrm{CS}$

11) Relative Accuracy (RA)

Assume calculation error multiplier $=\beta=10$ (few internal calculations and error introduced by data conversions)

Given $R A=\beta * \mathrm{Ra}_{\mathrm{a}}$ in $= \pm(014 \%)$ Full Range (for range $\left.40-140^{\circ} \mathrm{F}\right)$

$\mathrm{RA}= \pm 014 \% \mathrm{CS}$ (for range $40-140^{\circ} \mathrm{F}$ )

111) Static Pressure (SP)

$\mathrm{SP}=0 \% \mathrm{CS}$

Basis Not a pressure sensor, not subject to the pressure effects of the process

1v ) Humidity Effect (HE)

$\mathrm{HE}=0 \% \mathrm{CS}$

Basıs Housed in NEMA 4 enclosure

v) Temperature Effect (TE)

Given Reference accuracy is certıfied for range $40-140^{\circ} \mathrm{F}$

And design range is $40-115^{\circ} \mathrm{F}$ )

$\mathrm{TE}=0 \% \mathrm{CS}$

v1 ) Radiation Effect (RE)

Given Dose Rate $=85 \mathrm{mR} / \mathrm{hr}$

Total Integrated Dose $=85 \mathrm{mR} / \mathrm{hr} * 43800 \mathrm{hr}=372,300 \mathrm{mR}$

Radiation Effect Data says there is no effect to $10,000000 \mathrm{mR}$ TID

Because relatively small dose (based on HNF SD-SNF-DRD-002, Rev 1)

Assume $\mathrm{RE}= \pm 0 \% \mathrm{CS}$

v11 ) Power Supply Effect (PSE)

Given Vender information

No PSE while supply voltage maintained at $15 \mathrm{VDC} \pm 10 \%$ PSE $=0 \%$ 
MCO Vacuum transmitter

vi11) Seismic Effect (SE)

Device is qualıfied per IEEE 344-1987 to withstand $17 \mathrm{~g}$ seismic event

Design Basis event is postulated as $035 \mathrm{~g}$

$\mathrm{SE}=0 \%$

1X ) Sensor Measurement and Test Equipment Effect (SMTE)

Test Equipment1 Fluke 45, DC Volts, 5 digit display

$$
\begin{aligned}
\mathrm{RA}_{\mathrm{MTEl}} & =0025 \% \mathrm{Rdg}+2 \text { digits }=0025 \% \mathrm{CS}+002 \% \mathrm{CS} \\
& = \pm 0045 \% \mathrm{CS} \quad \text { (relative accuracy of MTE) }
\end{aligned}
$$

Note $0025 \%$ Rdg for a 100 torr reading is $0025 \%$ CS

$\mathrm{RA}_{\mathrm{STD} 1}=0 \% \mathrm{CS}$ (relative accuracy of standard)

$\mathrm{RD} 1=0 \% \mathrm{CS}$

$\mathrm{SA} 1=(0025 / 10) * 100=025 \% \mathrm{CS}$

$\mathrm{MTE} 1=\left(\mathrm{RA}_{\mathrm{MTE} 1}{ }^{2}+\mathrm{RA}_{\mathrm{STD} 1}{ }^{2}+\mathrm{RD}^{2}+\mathrm{SA}^{2}\right)^{1 / 2}$

MTE1 $=\left(0045^{2}+0^{2}+0^{2}+025^{2}\right)^{\prime}=0254 \% \mathrm{CS}$

$\mathrm{SMTE}=\mathrm{MTE} 1=0254 \% \mathrm{CS}$

x ) Total Uncertainties - Safety Class Trip Signal

$$
\begin{aligned}
\mathrm{e}^{+} & =\left[\mathrm{DR}^{2}+\mathrm{RA}^{2}+\mathrm{SP}^{2}+\mathrm{HE}^{2}+\mathrm{TE}^{2}+\mathrm{RE}^{2}+\mathrm{PSE}^{2}+\mathrm{SE}^{2}+\mathrm{SMTE}^{2}\right]^{1 / 2} \\
& =\left[002^{2}+014^{2}+0+0+0+0+0+0+0254^{2}\right]^{1 / 2}=0291 \% \mathrm{CS}
\end{aligned}
$$

The error associated with the processor $\mathrm{e}_{\mathrm{proc}}$ is as follows $\mathrm{e}_{\mathrm{proc}}= \pm 0291 \% \mathrm{CS}$

\section{Total Loop Uncertainty - 12 Torr Trip Signal Loop (SC)}

All bias terms are subtracted from the sensor (sens-dr) and processor (proc) error before combining terms then added back in

Safety class channel uncertainty (CU)

$$
\begin{aligned}
& \mathrm{CU}^{+}=\left[\mathrm{e}^{2} \text { sens } \mathrm{dr}+\mathrm{e}_{\text {proc }}^{2}\right]^{1 / 2}+\mathrm{DR}_{\text {sens }}+\mathrm{B}_{\text {proc }} \\
& \mathrm{CU}_{\mathrm{sc}}^{+}=\left[09^{2}+0291^{2}\right]^{1 / 2}+1+0=195 \% \mathrm{CS} \\
& \mathrm{CU}_{\mathrm{sc}}= \pm 195 \% \mathrm{CS}
\end{aligned}
$$

\section{Set Point Determınatıon}

A Trip Setpoint

The Analytical Limit (AL) is the maximum pressure allowable before the trip operates The Trip Setpoint (TS) TS $=\mathrm{AL}-(\mathrm{CU}+$ margin)

Given $A L=12$ Torr

Margin $=2$ torr (pressure drops inside the system 
MCO Vacuum transmitter

1) $\quad \mathrm{TS}_{\mathrm{sc}}=12-195-2=805$ Torr (safety class trip setpoint)

B Allowable Value

The allowable value (AV) provides a threshold value that can be used to assess the instruments expected performance when tested The Allowable Value setpoint trip margin (AVSTM) is the allowance between the trip setpoint and the AV

1)

$$
\begin{aligned}
& \mathrm{AV}=\mathrm{TS}+\left[\left(\mathrm{SMTE}^{2}+\mathrm{SMTE}^{2}+\mathrm{DR}_{\text {proc }}{ }^{2}\right)^{1 / 2}+\mathrm{DR}_{\text {sens }}\right]=\mathrm{TS}+\mathrm{AVSTM} \\
& \mathrm{AV}=805+\left(0355^{2}+0254^{2}+002^{2}\right)^{1 / 2}+1=805+044+1=949 \\
& \mathrm{AVSTM}=\mathrm{AV}-\mathrm{TS}_{\mathrm{sc}}=948-805=144
\end{aligned}
$$

\section{Delta Pressure Accuracy}

\section{Uncertainty Terms that Affect Accuracy}

Note Since this is a Delta Pressure over a relatıvely short tıme, several terms are no longer necessary

A Sensor Uncertainty

1) Relative Accuracy (RA)

Given $\mathrm{RA}=015 \%$ Reading (Rdg)

11) Drift (DR) Assume DR $=0$ for time interval

111 ) Framatome Input and Output Error

Processor Module input error calculation multiplier factor $\beta=10$

Accuracy $\pm 014 \%$ of Full Range (Analog Input)

$\pm 01 \%$ of Full Range (Analog Output)

Full Range $=10$ VDC $\Leftrightarrow 100$ torr

$$
\begin{aligned}
& \mathrm{RA}_{\mathrm{F}}=\left(\beta * \mathrm{RA}_{\mathrm{l}}^{2}+\mathrm{RA}_{\mathrm{O}}^{2}\right)^{\prime} \\
& \mathrm{RA}_{\mathrm{F}}=\left(014^{2}+01^{2}\right)^{\prime}=0172 \% \mathrm{CS}=017210 / 100^{*} 100 / 10=0172 \text { torr }
\end{aligned}
$$

1v ) Total Sensor Delta Pressure Uncertainties

Assume 2 hour Pressure Test with No Temperature Change $\Delta \mathrm{T}=0$

Calibration Temperature $\mathrm{Tc}=72^{\circ} \mathrm{F}=\mathrm{T}_{1}=\mathrm{T}_{2}$

Sensor and Readout Error on Span Measurements

Pressure rise from 5 to 74 torr

$$
\begin{aligned}
\mathrm{SE}_{1} & =+/-\left(\left[\left(\mathrm{RA}_{\mathrm{S}}\right)^{2}+\left(\mathrm{RA}_{\mathrm{F}}\right)^{2}\right]^{\prime}\right. \\
& =+/\left(\left[\left(00015^{*} 0\right)^{2}+(0172)^{2}\right]^{\prime}=+/ 0172\right. \text { torr } \\
\mathrm{SE}_{2} & =+/-\left(\left[\left(\mathrm{RA}_{\mathrm{S}}\right)^{2}+\left(\mathrm{RA}_{\mathrm{F}}\right)^{2}\right]^{\prime}\right. \\
& =+/-\left(\left[(00015 * 74)^{2}+(0172)^{2}\right]^{\prime}=+/ 0172\right. \text { torr }
\end{aligned}
$$


MCO Vacuum transmitter

For a pressure rise test from 50 torr to 74 torr, the error on each measurement is +/-0 17 torr Since it is a + / error, the maximum error would be 034 torr

1 e) $50+/ 017$ to $74+/-017$ becomes 483 to 757 or 517 to 723

Span $=24+/-034$ torr

$\mathrm{SE}_{\text {rтro }}=+/-034$ torr 


\section{MCO pressure transmitter to SCIC}

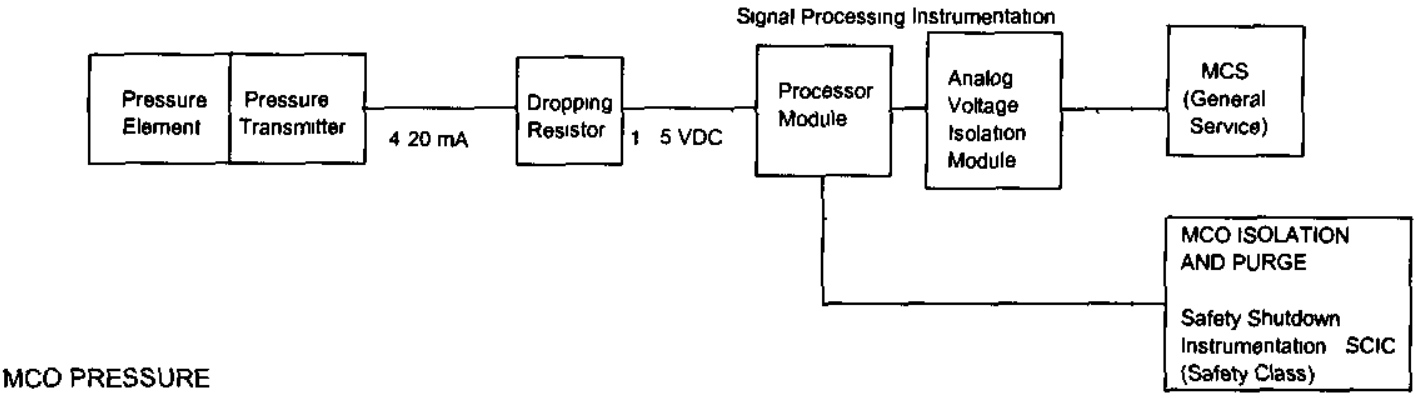

Figure 31 Rosemount Gauge Pressure Simplified Block Diagram

\section{Set Point Identification}

\section{Vacuum Trip}

Anytime during the DRYING mode (mode 5) the MCO pressure goes below $-117 \mathrm{psig}$ ( $\sim 155$ torr) then increases above $111 \mathrm{psig}$ ( $\sim 185$ torr) the MCS will automatically increase flow (FIT $1 * 20$ ) to $10 \mathrm{scfm}$ and re pressurıze the MCO to $\sim 10 \mathrm{psig}$ Restartıng a purge must satısfy the minımum purge rate of the parameter limit $(07 \mathrm{scfm})$ plus setpoint error Fallure to perform these steps will result in a SCIC MCO isolation and purge During the transition from above atmosphere to vacuum, time to reach less than $-117 \mathrm{psig}$ ( 155 torr) shall not exceed 5 minutes During the transition from below $-111 \mathrm{psig}(\sim 185$ torr) back to greater than 05 psig pressure, the time shall not exceed 5 minutes The MCO must be maintained above a positive pressure (approximately 05 psig) to prevent oxygen ingress unless a purge of adequate volume has been completed During bulk water drainıng, the MCO must remain above atmospherıc pressure If the MCO reaches an incorrect pressure state without adequate verified purge volume the SCIC will perform a MCO 1solation and purge

\section{MCO Low Pressure Trıp}

The safety function of the MCO LOW PRESSURE trip is prevent the ingress of air (oxygen) due to process system leaks The safety function of the SCIC, for this trip, is to actuate a $\mathrm{SC}$ isolation and SCHE purge prior to the MCO pressure dropping below atmospheric (preclude air ingress) Since the normal process cycles between pressure and vacuum this trip is mode dependent 


\section{Plant Operatıng Conditıons}

\section{Vacuum Trip - Accidents and responses}

During the transition from pressure operations to vacuum in MODES 5 and 6, the "MCO PRESSURE DECAY FAIL" trip is activated to ensure no leaks which would allow contınuous air cycling and to detect a degraded vacuum pump The MCO must reach the safety parameter limit of -114 psig within 5 minutes of entering vacuum ( $<024$ psig) Under normal MCS response in MODES 5, 6, and 7, flow will be re-started at $\sim 15 \mathrm{scfm}$ in response to being above 12 torr Once 12 torr is exceeded, the system pressure must go to $>024$ psig within 5 minutes of exceeding the safety parameter limit, timer initiation value of $-108 \mathrm{psig}$ In the event of a significant system leak pressure will not increase above 05 psig so within 5 minutes of exceeding the -111 psig ( 185 torr) setpoint value, the SCIC will cause an isolation and SCHe purge based on the "MCO PRESS RISE FAIL" trip

\section{Low Pressure Trip - Accidents and responses}

The MCO LOW PRESSURE trip will occur as soon as pressure drops below 024 psig and valve actuation will start within 03 seconds from this point The isolation valves take under a second to fully open (some testing has shown 05 seconds) but as soon as the valve comes off the closed seat, helium starts flowing to the $\mathrm{MCO}$

\section{Gauge Pressure Transmitter (PT-1*36, PT-1*37)}

The sensor chosen to monitor pressure for the $-117 \mathrm{psig}$ (155 torr), $-111 \mathrm{psig}$ (185 torr), and 05 psig analytical limits is a nuclear grade Gage Pressure Transmitters from Rosemount Nuclear This device is designed for nuclear safety class vacuum applications

\section{Instrument Data}

Name Pressure Transmitter

Manufacturer Rosemount Nuclear

Model Number $1153 \mathrm{~GB} 5$ ( $0-125$ to $0-750 \mathrm{in}_{2} \mathrm{O}$ )

Type Gauge Pressure Transmitter

\section{Reference Specifications (PDS 4302 April 1992 - Rosemount Nuclear)}

Range 0750 in $\mathrm{H}_{2} \mathrm{O}$

Resolution N/A (transmitter only)

Accuracy $\pm 025 \% \mathrm{CS}$

Input/Output 4-20 mA output

Calıbration Interval 1 year between calibrations

\section{Project Specifications}

Safety Classification Safety Class (SC)

Performance Category PC-3 for boundary only

Environmental Qualıfication Environmental Condition B

NPH Design Requirements Seismic Condition B

Required Safety Functions Pressure boundary integrity (signal is safety class non-se1smic) 
MCO Pressure Transmitter

334 Calıbration

A Assumptions

1) Calibrations performed at ambient $72 \pm 8^{\circ} \mathrm{F}$ or $64-80^{\circ} \mathrm{F}$

11) Calibration Interval $=1$ year

B Calculation Verification

1) Range

$\mathrm{URL}=750$ in $\mathrm{H}_{2} \mathrm{O}$

Zero $=147 \mathrm{psig}\left(-4069\right.$ in $\left.\mathrm{H}_{2} \mathrm{O}\right) \mathrm{Span}=12 \mathrm{psig}\left(33217\right.$ in $\left.\mathrm{H}_{2} \mathrm{O}\right) \mathrm{CS}=267$

psig (739 07 in $\left.\mathrm{H}_{2} \mathrm{O}\right)$

11 ) Turn Down Rat1o

$\mathrm{TDR}=\mathrm{URL} / \mathrm{CS}=750 / 739=1015$

111 ) Drift

$\pm 02 \%$ Upper Range Limit per 30 months

$\mathrm{DR}= \pm 02 \% *(\mathrm{URL} / \mathrm{CS})= \pm 02 \% \mathrm{CS}$

1v ) Relative Accuracy (RA)

Given $\mathrm{RA}= \pm 025 \% \mathrm{CS}$

v) Static Pressure (SP)

$\mathrm{SP}=0 \% \mathrm{CS}$

Basis $\pm 05 \%$ of $\mathrm{Rdg} / 1000 \mathrm{ps} 1$ It is systematic and can be calibrated out for a particular pressure before installation

v1 ) Humidity Effect (HE)

$\mathrm{HE}=0 \%$ Calibrated Span

Basıs $0-100 \%$ relative humidity (Housed in NEMA 4 enclosure)

v11 ) Temperature Effect (TE)

Given $\mathrm{TE}= \pm(075 \% \mathrm{URL}+05 \%$ span $)$ per $100^{\circ} \mathrm{F}$ ambient temperature change

$\mathrm{T}_{\text {cal }}=72 \pm 8^{\circ} \mathrm{F}$ (calibration temperature range)

$\mathrm{T}_{\min }=40^{\circ} \mathrm{F}$

$\mathrm{T}_{\max }=105^{\circ} \mathrm{F}$

Maxımum Tdelta $=\mathrm{T}_{\max }-\mathrm{T}_{\text {cal }}($ lower $)-=105^{\circ} \mathrm{F}-64^{\circ} \mathrm{F}=41$ delta $^{\circ} \mathrm{F}$

$\begin{aligned} \mathrm{TE} & = \pm[(075 \%) *(1013)+05 \%]^{*}(41 / 100) \\ & = \pm 0516 \mathrm{CS}\end{aligned}$

vili ) Radiation Effect (RE)

Given $\pm 80 \%$ URL at $22 \times 10^{7}$ rads TID 
Given Expected Dose Rate $=85 \mathrm{mR} / \mathrm{hr}$

$\mathrm{TID}=85 \mathrm{mR} / \mathrm{hr} * 43800 \mathrm{hr}=372,300 \mathrm{mR}=372 \times 10^{2} \mathrm{rads} \mathrm{TID}$ Approximate as zero because relatively small dose

$\mathrm{RE}= \pm 0 \% \mathrm{CS}$

1X ) Power Supply Effect (PSE)

Given $>0005 \%$ of output span/volt

PSE $>0001 \%$ CS

x ) Seismic Effect (SE)

No credit for this device is taken for a safety system shutdown during or after a seismic event

$\mathrm{SE}=0 \%$

x1 Sensor Measurement and Test Equipment Effect (SMTE)

Test Equipment 1 -Fluke 45, DC current mode $30 \mathrm{~mA}$ range with a 5 digit display Accuracy $=005 \% \mathrm{Rdg}+3 \mathrm{~d}$ igits

Max reading $=20 \mathrm{~mA}$

Calıbrated Span $=20-4=16 \mathrm{~mA}$

Relative accuracy of MTE1

$\mathrm{RA}_{\mathrm{MTE} 1}=005 \% * 20 / 16 \mathrm{CS}+(0003 \mathrm{~mA} / 16) * 100 \% \mathrm{CS}= \pm 008125 \% \mathrm{CS}$

$\mathrm{RA}_{\mathrm{STD} 1}=0 \% \mathrm{CS}$ (NIST standard is at least 4 times more accurate)

$\mathrm{RD} 1=0 \% \mathrm{CS}$ (digital readout)

$\mathrm{SA} 1=(0025 / 10)^{*} 100=025 \% \mathrm{CS}$ (assume tolerance of $\left.0025 \mathrm{URL}\right)$

$\mathrm{MTE} 1=\left(\mathrm{RA}_{\mathrm{MTE} 1}{ }^{2}+\mathrm{RA}_{\mathrm{STD1}}{ }^{2}+\mathrm{RD}^{2}+\mathrm{SA1}{ }^{2}\right)^{1 / 2}$

$\mathrm{MTE} 1=\left(008125^{2}+0^{2}+0^{2}+025^{2}\right)^{\prime}=0263$

Test Equipment2 Heise PTE 1 with HQS-2 sensor (0 30psig)

Accuracy $= \pm 01 \%$ span

Repeatability $= \pm 002 \%$ span

Temperature Effect $= \pm 0004 \%$ span per ${ }^{\circ} \mathrm{F}$

$\mathrm{CS}_{\text {test }}=30 \mathrm{ps} 1 \mathrm{~g}$

$\mathrm{CS}_{\text {Inst }}=267 \mathrm{psig}$

Relative accuracy of MTE2

$\mathrm{RA}_{\mathrm{MTE} 2}= \pm\left(\mathrm{Acc}^{2}+\text { Repeat }^{2}+\text { TempEffect }^{2}\right)^{1 / 2} * \mathrm{CS}_{\text {test }} / \mathrm{CS}_{\mathrm{Inst}}= \pm 018 \% \mathrm{CS}$

$\mathrm{RA}_{\mathrm{STD} 2}= \pm 002 \% \mathrm{CS}$

$\mathrm{RD} 2=0 \% \mathrm{CS}$ (digital readout)

$\mathrm{SA} 2=0 \% \mathrm{CS}$

$\mathrm{MTE} 2=\left(\mathrm{RA}_{\mathrm{MTE} 2}{ }^{2}+\mathrm{RA}_{\mathrm{STD} 2}{ }^{2}+\mathrm{RD} 2^{2}+\mathrm{SA} 2^{2}\right)^{1 / 2}$

$\mathrm{MTE} 2=\left(018^{2}+002^{2}+0^{2}+0^{2}\right)^{\prime}=018$

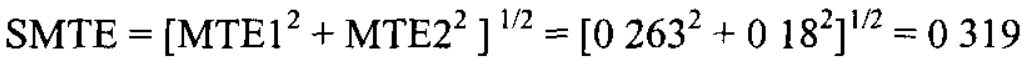


xi1 ) Total Sensor Uncertaintıes

$$
\begin{aligned}
& \mathrm{e}^{+}=\left[\mathrm{RA}^{2}+\mathrm{DR}^{2}+\mathrm{SP}^{2}+\mathrm{TE}^{2}+\mathrm{HE}^{2}+\mathrm{RE}^{2}+\mathrm{PSE}^{2}+\mathrm{SE}^{2}+\mathrm{SMTE}^{2}\right]^{\prime}+\mathrm{B} \\
& =\left[025^{2}+02^{2}+0+0516^{2}+0+0+0001^{2}+0+0319^{2}\right]^{1 / 2}+0=0686 \% \mathrm{CS} \\
& \mathrm{e}=-\left[\mathrm{RA}^{2}+\mathrm{DR}^{2}+\mathrm{SP}^{2}+\mathrm{TE}^{2}+\mathrm{HE}^{2}+\mathrm{RE}^{2}+\mathrm{PSE}^{2}+\mathrm{SE}^{2}+\mathrm{SMTE}^{2}\right]^{\prime}+\mathrm{B} \\
& =-\left[025^{2}+02^{2}+0+0516^{2}+0+0+0001^{2}+0+0319^{2}\right]^{1 / 2}+0=0686 \% \mathrm{CS} \\
& \text { sensor error }\left(\mathrm{e}_{\mathrm{sens}}\right) \\
& \mathrm{e}_{\text {sens }}= \pm 0686 \% \mathrm{CS} \\
& \mathrm{e}_{\text {sens }}= \pm(000686) * 267 \mathrm{psig}= \pm 018 \mathrm{psig}
\end{aligned}
$$

\section{Framatome STAR System}

\section{Processor Module}

See section 241

\section{Project Specifications}

See section 242

\section{Uncertainty terms that affect Calibration}

\section{A Assumptions}

1) Because of the random nature (direction and magnitude) of the drift, Calibration intervals of 12 months uses the same values as 6 months

11 ) Calibrations performed at ambient $72 \pm 8^{\circ} \mathrm{F}$ or $64-80^{\circ} \mathrm{F}$

111 ) $\mathrm{Min} / \mathrm{max}$ temperatures is $40-115^{\circ} \mathrm{F}$

1v ) Calibrated Span (CS) $=4$ VDC

v) Processor Module input error calculation multıplıer factor $=1$

B Calculation Verification Error to Safety Class Trip

1) Turn Down Ratio

$\mathrm{TDR}=\mathrm{URL} / \mathrm{CS}=10 / 4=25$

11) Drift (DR) DR $= \pm 005 \%$ CS (See section 244 )

1ii) Relative Accuracy (RA)

Assume calculation error multıplier $=\beta=10$

Given $R A=\beta * \mathrm{Ra}_{\mathrm{a} \text { in }}$

$$
= \pm(014 \%) \text { Full Range }
$$


MCO Pressure Transmitter

$\mathrm{RA}= \pm 035 \% \mathrm{CS}\left(\right.$ for $\mathrm{TDR}=25$ at $40-140^{\circ} \mathrm{F}$ )

1v ) Static Pressure (SP) SP $=0 \%$ CS (See section 24 4)

v) Humidity Effect (HE) $\mathrm{HE}=0 \% \mathrm{CS}$ (See section 244 )

v1 ) Temperature Effect (TE) $\mathrm{TE}=0 \% \mathrm{CS}$ (See section 244 )

vil ) Radiation Effect (RE) $\mathrm{RE}= \pm 0 \% \mathrm{CS}$ (See section 244 )

v111 ) Power Supply Effect (PSE) PSE = 0\% (See section 244 )

1X ) Seismic Effect (SE) SE $=0 \%$ (See section 244 )

x ) Sensor Measurement and Test Equipment Effect (SMTE)

Test Equipment1 Fluke 45 DC Volts, 5 digit display

$\mathrm{RA}_{\mathrm{MTEl}}=0$ 025\%Rdg +2 digits $=0025 \%+00004 \%$

$= \pm 00254 \% \mathrm{URL}(\mathrm{TDR})= \pm 00635 \% \mathrm{CS}$

(Note the $0004 \%$ is based on the 2 digits with a upper range limit of 5 VDC which is $00002 / 5) \times 100=0004 \%$ URL)

$\mathrm{RA}_{\mathrm{STDl}}=0 \% \mathrm{CS}$ (4 1 accuracy ratio)

$\mathrm{RD} 1=0 \% \mathrm{CS}$ (digital readout)

$\mathrm{SAl}=(0025) * 10=025 \% \mathrm{URL}=025 \%(\mathrm{TDR})=025 * 25=0625 \% \mathrm{CS}$

$\mathrm{MTE} 1=\left(\mathrm{RA}_{\mathrm{MTE}}{ }^{2}+\mathrm{RA}_{\mathrm{STD1}}{ }^{2}+\mathrm{RD}^{2}+\mathrm{SAl}^{2}\right)^{1 / 2}$

$\mathrm{MTE} 1=\left(00635^{2}+0^{2}+0^{2}+0625^{2}\right)^{\prime}=0628 \% \mathrm{CS}$

$\mathrm{SMTE}=\mathrm{MTE} 1=0628 \% \mathrm{CS}$

xı ) Total Uncertainties - Safety Class Trip Signal

$$
\begin{aligned}
\mathrm{e}^{+} & =\left[\mathrm{DR}^{2}+\mathrm{RA}^{2}+\mathrm{SP}^{2}+\mathrm{HE}^{2}+\mathrm{TE}^{2}+\mathrm{RE}^{2}+\mathrm{PSE}^{2}+\mathrm{SE}^{2}+\mathrm{SMTE}^{2}\right]^{\prime} \\
& =\left[005^{2}+035^{2}+0+0+0+0+0+0+0628^{2}\right]^{1 / 2}=0721 \% \mathrm{CS} \\
\mathrm{e}_{\mathrm{sc}} & = \pm 0721 \% \mathrm{CS} \\
\mathrm{e}_{\mathrm{sc}} & =0721 / 100^{*} 4= \pm 0029 \mathrm{VDC}
\end{aligned}
$$




\section{Total Loop Uncertainty - Safety Class Loop}

Sensor (sens) and processor (proc) loop error

$\mathrm{CU}^{+}=\left[\mathrm{e}_{\text {sens }}^{2}+\mathrm{e}_{\text {proc }}^{2}\right]^{1 / 2}+\mathrm{B}_{\text {sens }}+\mathrm{B}_{\text {proc }}$

$\mathrm{CU}_{\mathrm{sc}}^{+}=\left[0686^{2}+0721^{2}\right]^{1 / 2}+0+0=0995 \% \mathrm{CS}$

Channel Uncertanty (CU)

$\mathrm{CU}_{\mathrm{sc}}= \pm 0993 \% \mathrm{CS}$

$\mathrm{CU}_{\mathrm{sc}}= \pm 000993 * 267= \pm 0265 \mathrm{ps} \mathrm{g}$

\section{Set Point Determination}

A Trip Setpoint

The Analytical Limit (AL) is the maximum pressure allowable before the trip operates The Trip Setpoint $(\mathrm{TS}) \mathrm{TS}=\mathrm{AL}-(\mathrm{CU}+$ margin)

Given $\mathrm{AL}_{1}=-114 \mathrm{psig}$ (with a margin of zero)

$\mathrm{AL}_{2}=109 \mathrm{psig}$ (with a margin of zero)

$\mathrm{AL}_{3}=05 \mathrm{psig}$ (with a margin of zero)

1) $\mathrm{TS}_{\mathrm{scl}}=-114-0265 \mathrm{psig}=-11665 \mathrm{psig}$

11) $\quad \mathrm{TS}_{\mathrm{sc} 2}=-108 \quad 0265 \mathrm{psig}=-11065 \mathrm{ps} 1 \mathrm{~g}$

111 ) $\quad \mathrm{TS}_{\mathrm{sc} 3}=05+0265 \mathrm{psig}=0765 \mathrm{psig}$

$$
=05-0265 \mathrm{psig}=0235 \mathrm{psig}
$$

B Allowable Value

The allowable value (AV) provides a threshold value that can be used to assess the instruments expected performance when tested The allowance between the trip setpoint and the AV includes Drift, instrument calibration uncertainties and any additional instrument uncertainties durnng normal operation that are measured during testing The purpose for determining an AV is to provide a threshold value that can be used to assess the instrument $s$ performance when tested The Allowable Value setpoint trip margin (AVSTM)

1) $\left.\mathrm{AV}=\mathrm{TS}+/-\left[\left(\mathrm{SMTE}^{2}+\mathrm{SMTE}^{2}+\mathrm{DR}_{\text {sens }^{2}}+\mathrm{DR}_{\text {proc }}\right)^{2}\right)^{1 / 2}\right]=\mathrm{TS}+/ \mathrm{AVSTM}$

$$
\begin{aligned}
& \mathrm{AV}=\mathrm{TS}-\left(0319^{2}+0635^{2}+01^{2}+005^{2}\right)^{1 / 2} * 267 / 100 \\
& \mathrm{AV}=\mathrm{TS}-0719 * 267 / 100=\mathrm{TS}-0192 \mathrm{psig} \\
& \mathrm{AVSTM}=0192 \mathrm{psig}
\end{aligned}
$$




\section{Temperature switch / transmitter to SCIC}

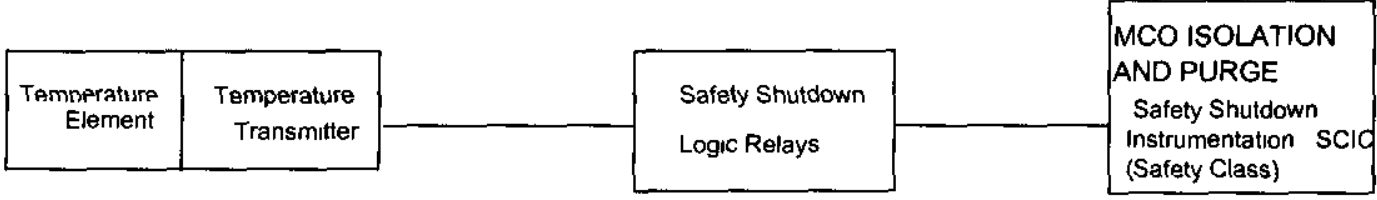

PROCESS

Figure 41 Temperature Switch / Transmitter Simplıfied Block Diagram

\section{Set Point Identification}

\section{TW Annulus Water Temperature}

The $50^{\circ} \mathrm{C}$ TW Annulus Water Temperature parameter limit value is required for fuel cooling and is identified in the CVDF SAR This trip results in contacts opening in the SCIC to deenergize the Tempered Water (TW) heater starter The TW heater are normally controlled by the MCS through a separate starter box, power then is routed through the Train A then Train B starters Unless a fault condition (high temperature) occurs these SCIC starters remain closed allowing normal MCS control This caused the 480VAC to be removed, thus de-energizing the water heater

\section{High Bay Temperature}

A $95^{\circ} \mathrm{F}$ bay high temperature parameter limit value is required because of the current fire hazards analysis Additionally, a $105^{\circ} \mathrm{F}$ bay high temperature parameter limit value is required to mitigate impacts to safety-class instrument calibration $105^{\circ} \mathrm{F}$ is the operational limit of the safety class Baratron pressure sensors A high process bay temperature initiates an SCIC system trip that isolates the $\mathrm{MCO}$ and activates the SCHe system, thereby pressurizing and purging the $\mathrm{MCO}$

\section{Plant Operatıng Conditıons}

\section{TW Annulus Water Temperature}

If a high TW (annulus) trip occurs during processing, the SCIC system shuts down the water heater These actions are fully automatic and require no operator action

\section{High Bay Temperature}

A high process bay temperature initiates an SCIC system ISO\&PURGE These actions are fully automatic and require no operator action 
Temperature Switch / Transmitter

SNF-4451 Rev 1 Page 26 of 50

\section{Temperature Switch / Transmitter (TSH-1*28, 1*29, TSHH-1*38, 1*39)}

\section{Instrument Data}

Name Temperature Switch

Manufacturer Ashcroft

Model Number LTDN4KK00040

Type Temperature Switch / Transmitter

432 Reference Specifications (SW-12-Ashcroft)

Range $20-95^{\circ} \mathrm{C}\left(68-203^{\circ} \mathrm{F}\right) \quad$ (SW 12 - order chorce - Ashcroft)

Repeatability $\pm 1 \%$ span set point repeatability (SW/PI-62 4/3/95 - Ashcroft)

Accuracy $\pm 1 \%$ span (assume same as repeatability)

Calibration Interval 1 month

\section{Project Specifications}

Safety Classıfication Safety Class (SC)

Performance Category PC-1

Environmental Qualification Environmental Conditıon B

NPH Design Requirements NA

Safety Functions TSH-1*28,1*29 TW isolation for H2 Explosion prevention, Runaway Reaction Prevention (non-seismic)

TSH-1*38, 1*39 Instrument Environmental Monitoring (temperature) for $\mathrm{H} 2$ Explosion prevention (non seismic)

434 Calıbration

A Assumptions

1) Calıbrations performed at ambient $72 \pm 8^{\circ} \mathrm{F}$ or $64-80^{\circ} \mathrm{F}$

11) $\mathrm{Min} / \mathrm{max}$ temperature range $1 \mathrm{~s} 40-115^{\circ} \mathrm{F}$

111 ) Calibration Interval (TSH-1*28, $1 * 29)=3$ month

iv ) Calibration Interval (TSH-1*38 1*39) = 1 year

B Calculation Verification

1) Range

$\mathrm{URL}=95^{\circ} \mathrm{C}$

Zero $=20^{\circ} \mathrm{C}$

Span $=75^{\circ} \mathrm{C}$

$\mathrm{CS}=75^{\circ} \mathrm{C}$

11) Turn Down Ratio

$\mathrm{TDR}=1$ (F1xed range no TDR required) 
111 ) Drift

$\pm 1 \%$ Span per month

DR (TSH- $1 * 281 * 29)= \pm(1 \% * 3 \text { months })^{1 / 2}= \pm 173 \% \mathrm{CS}$

DR (TSH- $1 * 38,1 * 39)= \pm(1 \% * 12 \text { months) })^{1 / 2}= \pm 346 \%$ CS

1v) Relative Accuracy (RA)

Given Accuracy $= \pm 1 \% \mathrm{CS}$ and Repeatability $= \pm 1 \% \mathrm{CS}$

$\mathrm{RA}= \pm(1 \%+1 \%)^{1 / 2}$

$\mathrm{RA}= \pm 14 \%$

v) Static Pressure (SP)

$\mathrm{SP}=0 \% \mathrm{CS}$

Basis Temperature element is isolated from the process or exposed to environments with atmospheric pressure expected

v1 Humidity Effect (HE)

$\mathrm{HE}=0 \%$ Cahbrated Span

Basis Water tight and corrosion resistant (Housed in NEMA 4,4X enclosure)

vi1 ) Temperature Effect (TE)

Given $\mathrm{TE}= \pm\left(1 \%\right.$ span per $50^{\circ} \mathrm{F}$ ambient temperature change

$\mathrm{T}_{\text {cal }}=72 \pm 8^{\circ} \mathrm{F}$ (calibration temperature range)

$\mathrm{T}_{\mathrm{min}}=40^{\circ} \mathrm{F}$

$\mathrm{T}_{\max }=115^{\circ} \mathrm{F}$

Maxımum Tdelta $=\mathrm{T}_{\max }-\mathrm{T}_{\text {cal }}($ lower $)=115^{\circ} \mathrm{F}-64^{\circ} \mathrm{F}=51 \mathrm{delta}^{\circ} \mathrm{F}$

$\mathrm{TE}= \pm 1 \% \mathrm{CS}$

vi11 ) Radiation Effect (RE)

Given $\pm 80 \% \mathrm{URL}$ at $22 \times 10^{7} \mathrm{rads}$ TID

Given Expected Dose Rate $=85 \mathrm{mR} / \mathrm{hr}$

TID $=85 \mathrm{mR} / \mathrm{hr} * 43800 \mathrm{hr}=372,300 \mathrm{mR}=372 \times 10^{2} \mathrm{rads}$ TID

Approximate as zero because of mild dose

$\mathrm{RE}= \pm 0 \% \mathrm{CS}$

1x ) Power Supply Effect (PSE)

Assume

$\mathrm{PSE}=0 \% \mathrm{CS}$

x ) Se1smic Effect (SE)

No credit for this device is taken for a safety system shutdown during or after a seismic event

$\mathrm{SE}=0 \%$ 
x1 ) Sensor Measurement and Test Equipment Effect (SMTE)

Test Equipment1 -AMETEK 140SE JOFRA dry block

Accuracy $= \pm 03{ }^{\circ} \mathrm{C}$

Insertion Depth Effect $= \pm 01^{\circ} \mathrm{C}$

$\mathrm{RA}_{\mathrm{MTE1}}=$ Accuracy + Insertion Depth Effect

$\mathrm{RA}_{\mathrm{MTE} 1}=03+01=04{ }^{\circ} \mathrm{C}=04 / 75 \mathrm{CS}=053 \% \mathrm{CS}$

$\mathrm{RA}_{\mathrm{MTE} 1}= \pm 053 \% \mathrm{CS}$

$\mathrm{RA}_{\text {STDI }}= \pm 01{ }^{\circ} \mathrm{C}$ (NIST standard (RTD used for calıbration)

$\mathrm{RA}_{\text {STD } 1}=01{ }^{\circ} \mathrm{C}(100 / 75)=01 \% \mathrm{CS}$

$\mathrm{RD} 1=0 \% \mathrm{CS}$ (digital readout)

$\mathrm{SA} 1=01 \% \mathrm{CS}$

(Assume standard temperature setpoint accuracy equal to relative accuracy)

$$
\begin{aligned}
& \mathrm{MTE} 1=\left(\mathrm{RA}_{\mathrm{MTE1}}{ }^{2}+\mathrm{RA}_{\mathrm{STD} 1}{ }^{2}+\mathrm{RD}^{2}+\mathrm{SA}^{2}+\mathrm{SA}^{2}\right)^{1 / 2} \\
& \mathrm{MTE} 1=\left(053^{2}+01^{2}+0+01^{2}\right)^{\prime}=055 \% \mathrm{CS}
\end{aligned}
$$

xi1 ) Setpoint setting accuracy

$\mathrm{SA}= \pm 10 \% \mathrm{CS}$

(Assume Ashcroft temperature setpoint setting accuracy is equal to the sensor relatıve accuracy)

xi11 ) Total Uncertaintıes

(TSH-1*28 1*29)

$$
\begin{aligned}
& \mathrm{e}^{+}=\left[\mathrm{RA}^{2}+\mathrm{DR}^{2}+\mathrm{SP}^{2}+\mathrm{TE}^{2}+\mathrm{HE}^{2}+\mathrm{RE}^{2}+\mathrm{PSE}^{2}+\mathrm{SE}^{2}+\mathrm{SMTE}^{2}+\mathrm{SA}^{2}\right]^{\prime}+\mathrm{B} \\
& \quad=\left[\begin{array}{ll}
1 & \left.0^{2}+173^{2}+0+1^{2}+0+0+0+0+055^{2}+10^{2}\right]^{1 / 2}+0=25 \% \mathrm{CS} \\
\mathrm{e} & =-\left[\begin{array}{ll}
1 & 0^{2}+173^{2}+0+1^{2}+0+0+0+0+055^{2}+10^{2}
\end{array}\right]^{1 / 2}+0=-25 \% \mathrm{CS}
\end{array}\right. \\
& \mathrm{e}_{\text {sens_l } 28}= \pm 25 \% \mathrm{CS}= \pm(0025 * 75)= \pm 188^{\circ} \mathrm{C}
\end{aligned}
$$

(TSH-1*38, 1*39)

$$
\begin{aligned}
& \mathrm{e}^{+}=\left[\mathrm{RA}^{2}+\mathrm{DR}^{2}+\mathrm{SP}^{2}+\mathrm{TE}^{2}+\mathrm{HE}^{2}+\mathrm{RE}^{2}+\mathrm{PSE}^{2}+\mathrm{SE}^{2}+\mathrm{SMTE}^{2}+\mathrm{SA}^{2}\right]^{\prime}+\mathrm{B} \\
& \quad=\left[\begin{array}{lll}
1 & \left.41^{2}+35^{2}+0+1^{2}+0+0+0+0+055^{2}+10^{2}\right]^{1 / 2}+0=4067 \% \mathrm{CS} \\
\mathrm{e} & =-\left[\begin{array}{lll}
1 & 41^{2}+35^{2}+0+1^{2}+0+0+0+0+055^{2}+10^{2}
\end{array}\right]^{1 / 2}+0=-4067 \% \mathrm{CS}
\end{array}\right. \\
& \mathrm{e}_{\text {sens } 1 * 38}= \pm 4067 \% \mathrm{CS}= \pm(00467 * 75)= \pm 305^{\circ} \mathrm{C}
\end{aligned}
$$

xiv ) Channel Uncertainty (CU)

(TSH $1 * 28,1 * 29)$

$\mathrm{CU}_{1 * 28}= \pm 25 \% \mathrm{CS}$ (calibration interval 3 months)

$\mathrm{CU}_{1 * 28}= \pm 25 / 100 * 75= \pm 188^{\circ} \mathrm{C}$ 


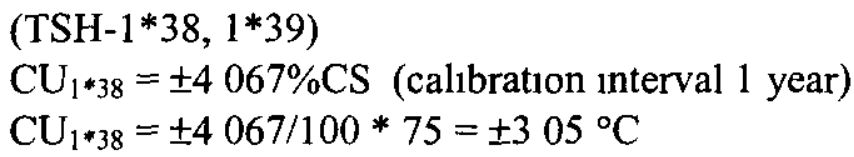

\section{Set Point Determination}

A Trip Setpoint - TW Annulus Water Temperature The Analytical Limit (AL) is the maximum temperature allowable before the trip operates The Trup Setpoint (TS)

Given $\mathrm{AL}=50^{\circ} \mathrm{C}$ (with a margin of zero)

1) $\mathrm{TS}=\mathrm{AL}-(\mathrm{CU}+$ margin $)$

$\mathrm{TS}_{\mathrm{sc} \mathrm{I} * 28}=50-(188+0)=4812^{\circ} \mathrm{C}$ (Calibration interval 3 months)

B Allowable Value

The allowable value (AV) provides a threshold value that can be used to assess the instruments expected performance when tested The purpose for determining an AV is to provide a threshold value that can be used to assess the instrument $\mathrm{s}$ performance when tested The Allowable Value setpoint trip margin (AVSTM)

1) $\quad \mathrm{AV}_{\mathrm{sc} 1^{* 28}}=\mathrm{TS}_{\mathrm{sc}}+\left(\mathrm{SMTE} 1^{2}+\mathrm{DR}^{2}\right)^{1 / 2}=\mathrm{TS}_{\mathrm{sc}}+\mathrm{AVSTM}$

$\mathrm{AV}_{\mathrm{sc} 1 * 28}=4812+\left(055^{2}+173^{2}\right)^{1 / 2} 75 / 100=4812+182^{*} 75 / 100=4948^{\circ} \mathrm{C}$ (calibration 3 months)

C Trip Setpoint - High Bay Temperature

The Analytical Limit (AL) is the maximum pressure allowable before the trip operates The Trip Setpoint (TS)

Given $\mathrm{AL}=95^{\circ} \mathrm{F}$ (with a margin of zero)

$\mathrm{CU}=305^{\circ} \mathrm{C}=549^{\circ} \mathrm{F}$

11) $\mathrm{TS}=\mathrm{AL}-(\mathrm{CU}+$ margin $)$

$\mathrm{TS}_{\mathrm{sc} 1 * 38}=95-549=8951^{\circ} \mathrm{F}$ (Calibration interval 1 year $)$

D Allowable Value

The allowable value (AV) provides a threshold value that can be used to assess the instruments expected performance when tested The purpose for determining an AV is to provide a threshold value that can be used to assess the instrument s performance when tested The Allowable Value setpoint trip margin (AVSTM)

11) $\quad \mathrm{AV}_{\mathrm{sc}}=\mathrm{TS}_{\mathrm{sc}}+\left(\mathrm{SMTE} 1^{2}+\mathrm{DR}^{2}\right)^{1 / 2}=\mathrm{TS}_{\mathrm{sc}}+\mathrm{AVSTM}$ $\mathrm{AV}_{\mathrm{sc}}=8951+\left(055^{2}+35^{2}\right)^{1 / 2} 75 / 100=8951+266=9217^{\circ} \mathrm{F}$ (callbration 1 year) 
5 Minımum Purge Flow transmitter to SCIC

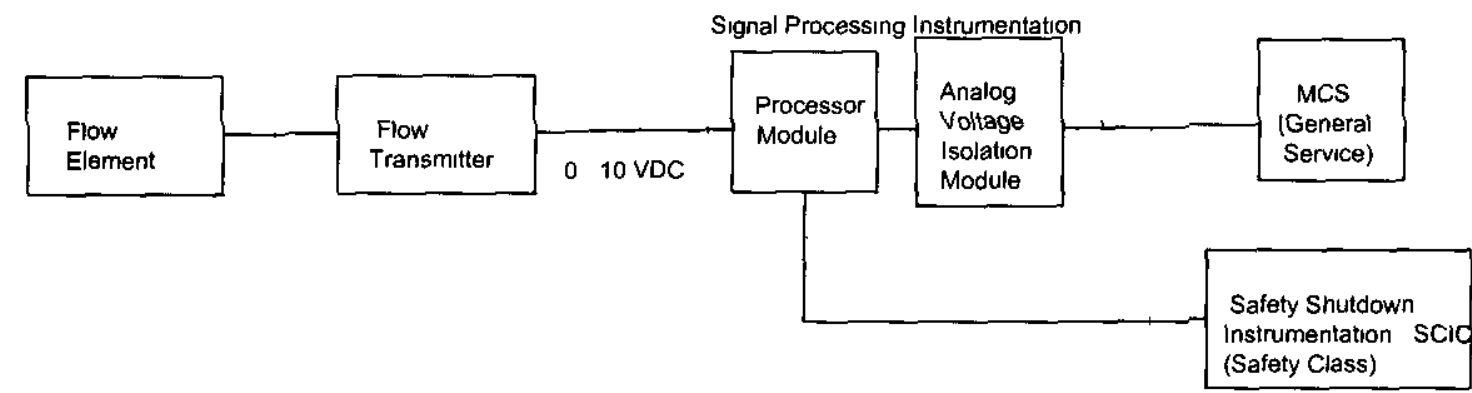

HELIUM Flow

MCO ISOLATION AND PURGE

Figure 51 FCI Mass Flow Transmitter Simplified Block Diagram

\section{Set Point Identification}

\section{PWC Low Purge Flow Alarm}

This alarm signifies that, during the PWC Pre-purge or Post-purge either there was a lowflow rate or the minımum time for the purge was not met Manual operator action is required upon an alarm This alarm function is defense-in-depth

\section{Mass Flow Transmitter (FIT-1*20, FIT-1*21)}

\section{Instrument Data}

Name Mass Flow Meter

Manufacturer Fludd Components Inc

Model Number LT87 (11E000004A2BA00)

Type Mass Flow Transmitter

522 Reference Specifications (Specification 12/95- FCI Fluid Components, Inc )

Range $0-10 \mathrm{scfm}$

Repeatability $05 \%$ Rdg

Accuracy $\pm 1 \%$ Full Scale

Input/Output $0-10$ Volt output

Calibration Interval 1 year between calibrations 
MCO Flow Transmitter

\section{Project Specifications}

Safety Classification Safety Class (SC)

Performance Category PC-1

Environmental Qualification Environmental Condition B

NPH Design Requirements N/A

Required Safety Functions Thermal Runaway and H2 Explosion prevention Provides SCIC trip inputs Pressure boundary integrity (signal is non-seismic)

\section{Calibration}

\section{A Assumptions}

1) Calibrations performed at ambient $72 \pm 8^{\circ} \mathrm{F}$ or $64-80^{\circ} \mathrm{F}$

11) $\mathrm{Min} / \max$ temperature range $1 \mathrm{~s} 40-115^{\circ} \mathrm{F}$

111 ) Calibration Interval $=1$ year

B Calculation Verification

1) Range

Zero $=0 \mathrm{scfm}$

Span $=10 \mathrm{scfm}$

$\mathrm{CS}=10 \mathrm{scfm}$

$\mathrm{URL}=10 \mathrm{scfm}$

11 ) Turn Down Ratio

$\mathrm{TDR}=\mathrm{URL} / \mathrm{CS}=1$

111 ) Drift (Qualification Report \#708349 - FCI Fluid Components, Inc )

$\pm 22 \%$ Full Scale (FS) per year

$\mathrm{DR}= \pm 22 \% \mathrm{CS}$

1v) Relative Accuracy (RA)

Given $\mathrm{RA}= \pm 3 \% \mathrm{FS}$ for helium (letter from $\mathrm{H}$ Sittınger App Engineer FCI) $\mathrm{RA}= \pm 3 \% \mathrm{CS}$

v) Statıc Pressure (SP) (Qualıfication Report \#708349 - FCI, Inc )

$\mathrm{SP}= \pm 1 \% \mathrm{FS} / 50 \mathrm{psig}$

$\mathrm{SP}=($ Max pressure $)($ Static pressure effect $)$

$\mathrm{SP}=(20 \mathrm{ps} 1 \mathrm{~g})( \pm 1 \% \mathrm{FS} / 50 \mathrm{ps} 1 \mathrm{~g})$

$\mathrm{SP}= \pm 04 \% \mathrm{CS})$ Note This is listed as a bias

v1 Humidity Effect (HE)

$\mathrm{HE}=0 \%$ Calibrated Span

Bası $0-100 \%$ relative humidity (Housed in NEMA 4 enclosure) 
v11) Temperature Effect (TE)

Given $\mathrm{TE}= \pm 0 \% \mathrm{CS}$

Basis This device uses temperature compensation The stated accuracy is maintained for a process temperature range of $\pm 250^{\circ} \mathrm{F} \mathrm{Min} / \mathrm{max}$ expected temperature range is $40-115^{\circ} \mathrm{F}$

viii ) Radiation Effect (RE)

Given $\pm 80 \%$ URL at $22 \times 10^{7}$ rads TID

Given Expected Dose Rate $=85 \mathrm{mR} / \mathrm{hr}$

TID $=85 \mathrm{mR} / \mathrm{hr} * 43800 \mathrm{hr}=372300 \mathrm{mR}=372 \times 10^{2}$ rads TID

Approxımate as zero because relatively small dose

$\mathrm{RE}= \pm 0 \% \mathrm{CS}$

1x ) Power Supply Effect (PSE)

Assume PSE $= \pm 0 \%$ CS

x) Seismic Effect (SE)

No credit for this device is taken for a safety system shutdown during or after a seismic event

$\mathrm{SE}= \pm 0 \%$

x1 ) Sensor Measurement and Test Equipment Effect (SMTE)

Test Equipment1 -FCI calibration laboratory instrumentation

Accuracy $=07 \% \operatorname{Rdg}$ (NIST Traceable)

Max reading $=10 \mathrm{scfm}$

Calibrated Span $=10 \mathrm{scfm}$

$\mathrm{SMTE}= \pm 07 \% \mathrm{CS}$ (worst case)

x11 ) Total Uncertainties

$$
\begin{aligned}
& \begin{aligned}
\mathrm{e}^{+} & =\left[\mathrm{RA}^{2}+\mathrm{DR}^{2}+\mathrm{TE}^{2}+\mathrm{HE}^{2}+\mathrm{RE}^{2}+\mathrm{PSE}^{2}+\mathrm{SE}^{2}+\mathrm{SMTE}^{2}\right]^{1 / 2}+\mathrm{SP} \\
& =\left[3^{2}+22^{2}+0+0^{2}+0+0+0^{2}+0+07^{2}\right]^{1 / 2}+04=3785 \% \mathrm{CS} \\
\mathrm{e} & =\left[\mathrm{RA}^{2}+\mathrm{DR}^{2}+\mathrm{TE}^{2}+\mathrm{HE}^{2}+\mathrm{RE}^{2}+\mathrm{PSE}^{2}+\mathrm{SE}^{2}+\mathrm{SMTE}^{2}\right]^{\prime}+\mathrm{SP} \\
& =\left[3^{2}+22^{2}+0+0^{2}+0+0+0^{2}+0+07^{2}\right]^{1 / 2}+04=3785 \% \mathrm{CS}
\end{aligned} \\
& \mathrm{e}_{\text {sens }}= \pm 3785 \% \mathrm{CS}= \\
& \mathrm{e}_{\text {sens }}= \pm(003785)^{*} 10 \mathrm{scfm}= \pm 03785 \mathrm{scfm}
\end{aligned}
$$


MCO Flow Transmitter

SNF-4451 Rev 1 Page 33 of 50

\section{Framatome STAR System}

\section{Processor Module}

$5311 \quad$ Instrument Data

Name Processor Module

Manufacturer Framatome Technologies

Model Number 1225275-002

Type Signal Processing Module

5312 Reference Specifications (1960501 5/95 - Framatome)

Range 0 to $10 \mathrm{VDC}$

Resolution 14 bits (DAC)

Drift \pm 2 mVDC $= \pm 002 \%$ Full Scale

Accuracy $\pm 014 \%$ of Full Range (Analog Input)

$\pm 01 \%$ of Full Range (Analog Output)

Analog Input/Output Range $00 \mathrm{VDC}$ to $100 \mathrm{VDC}$

Calibration Interval 12 months between calıbrations

\section{Project Specifications}

Safety Classification Safety Class (SC)

Performance Category PC-3

Environmental Qualification Environmental Condition B

NPH Design Requirements Seismic Condition C

Required Safety Functions H2 Explosion prevention, Runaway Reaction Prevention

\section{Calıbration}

A Assumptions

1) Turn Down Ratio $=1$

11) Calıbrations performed at ambient $72 \pm 8^{\circ} \mathrm{F}$ or $64-80^{\circ} \mathrm{F}$

111 ) $\mathrm{Min} / \mathrm{max}$ expected temperatures is $40-95^{\circ} \mathrm{F}$

iv ) Calibrated Span (CS) $=$ Full Scale $=10 \mathrm{VDC}$

v) Processor Module input error calculation multıplıer factor $=1$

B Channel Uncertainty Safety Class Trip Signal
1) Drift (DR)
$\mathrm{DR} 1=002$
$\mathrm{DR}= \pm 002 \% \mathrm{CS}$ 
11) Relative Accuracy (RA)

Assume calculation error multıplier $=\beta=10$

Given $\mathrm{RA}=\beta * \mathrm{Ra}_{\mathrm{a}}$ in

$$
= \pm(014 \%) \text { Full Range (for range } 40-140^{\circ} \mathrm{F} \text { ) }
$$

$\mathrm{RA}= \pm 014 \% \mathrm{CS}$ (for range $40-140^{\circ} \mathrm{F}$ )

111 ) Static Pressure (SP)

$\mathrm{SP}=0 \% \mathrm{CS}$

Basis Not a pressure sensor, not subject to the pressure effects of the process

1v ) Humidity Effect (HE)

$\mathrm{HE}=0 \% \mathrm{CS}$

Basis Housed in NEMA 4 enclosure

v) Temperature Effect (TE)

Given Reference accuracy is certified for range $40-140^{\circ} \mathrm{F}$

And expected range $40-95^{\circ} \mathrm{F}$ )

$\mathrm{TE}=0 \% \mathrm{CS}$

vi ) Radiation Effect (RE)

Given Dose Rate $=85 \mathrm{mR} / \mathrm{hr}$

Total Integrated Dose $=85 \mathrm{mR} / \mathrm{hr} * 43800 \mathrm{hr}=372,300 \mathrm{mR}$

Radiation Effect Data says there is no effect to $10,000,000 \mathrm{mR}$ TID

Because relatıvely small dose (based on HNF-SD-SNF-DRD-002, Rev 1)

Assume $\mathrm{RE}= \pm 0 \% \mathrm{CS}$

vil ) Power Supply Effect (PSE)

Given Vender information

No PSE while supply voltage maintained at $15 \mathrm{VDC} \pm 10 \%$

$\mathrm{PSE}=0 \%$

v111) Se1smic Effect (SE)

Device is qualified per IEEE $344-1987$ to withstand $17 \mathrm{~g}$ seismic event

Design Basis event is postulated as $035 \mathrm{~g}$

$\mathrm{SE}=0 \%$

1x ) Sensor Measurement and Test Equipment Effect (SMTE)

Test Equipment1 Fluke 45, DC Volts, 5 digit display

$\mathrm{RA}_{\mathrm{MTE} 1}=0025 \% \mathrm{Rdg}+2 \mathrm{digits}=0025 \%+0002 \mathrm{VDC} / 10 \mathrm{VDC} * 100$

$$
= \pm 0045 \% \mathrm{CS}
$$

$\mathrm{RA}_{\mathrm{STD} 1}=0 \% \mathrm{CS}$

$\mathrm{RD} 1=0 \% \mathrm{CS}$

$\mathrm{SA} 1=(0025 / 10)^{*} 100=025 \% \mathrm{CS}$ 
MCO Flow Transmitter

$$
\begin{aligned}
& \text { MTE1 }=\left(\mathrm{RA}_{\mathrm{MTE}}{ }^{2}+\mathrm{RA}_{\mathrm{STD1}}{ }^{2}+\mathrm{RD}^{2}+\mathrm{SAl}^{2}\right)^{1 / 2} \\
& \text { MTE1 }=\left(0045^{2}+0^{2}+0^{2}+025^{2}\right)^{\prime}=0254 \% \mathrm{CS} \\
& \text { SMTE }=\text { MTE1 }=0254 \% \mathrm{CS}
\end{aligned}
$$

x ) Total Uncertainties - Safety Class Trip Signal

$$
\begin{aligned}
\mathrm{e}^{+} & =\left[\mathrm{DR}^{2}+\mathrm{RA}^{2}+\mathrm{SP}^{2}+\mathrm{HE}^{2}+\mathrm{TE}^{2}+\mathrm{RE}^{2}+\mathrm{PSE}^{2}+\mathrm{SE}^{2}+\mathrm{SMTE}^{2}\right]^{\prime} \\
& =\left[\begin{array}{lll}
0 & \left.02^{2}+014^{2}+0+0+0+0+0+0+0254^{2}\right]^{1 / 2}=0291 \% \mathrm{CS}
\end{array}\right. \\
\mathrm{e}_{\mathrm{sc}} & = \pm 0291 \% \mathrm{CS}
\end{aligned}
$$

\section{Total Loop Uncertainty - Safety Class Trip Signal Loop}

$$
\begin{aligned}
& \mathrm{CU}^{+}=\left[\mathrm{e}_{\text {sens dr }}^{2}+\mathrm{e}_{\text {proc }}^{2}\right]^{1 / 2}+\mathrm{B}_{\text {sens }}+\mathrm{B}_{\text {proc }} \\
& \mathrm{CU}_{\mathrm{sc}}^{+}=\left[3785^{2}+0291^{2}\right]^{1 / 2}+014+0=393 \% \mathrm{CS} \\
& \mathrm{CU}_{\mathrm{sc}}= \pm 393 \% \mathrm{CS} \\
& \mathrm{CU}_{\mathrm{sc}}= \pm 00393 * 10= \pm 0393 \mathrm{scfm}
\end{aligned}
$$

\section{Set Point Determınation}

A Trip Setpoint

The Analytical Limit (AL) is the minimum flow allowable before the trip operates The Trip Setpoint (TS)

Given $\mathrm{AL}=070 \mathrm{scfm}$ (with a margin of zero)

$$
\begin{aligned}
& \mathrm{TS}=\mathrm{AL}+(\mathrm{CU}+\text { margin) } \\
& \mathrm{TS}_{\mathrm{sc}}=070+039=109 \mathrm{scfm} \text { (round to } 11 \text { ) }
\end{aligned}
$$

B Allowable Value

The allowable value (AV) provides a threshold value that can be used to assess the instruments expected performance when tested The allowance between the trip setpoint and the AV includes Drift, instrument calibration uncertainties, and any additional instrument uncertaintıes during normal operation that are measured during testing The purpose for determining an AV is to provide a threshold value that can be used to assess the instrument s performance when tested The Allowable Value setpoint trip margin (AVSTM)

11) $\quad \mathrm{AV}_{\mathrm{sc}}=\mathrm{TS}_{\mathrm{sc}}-\left(\mathrm{SMTE}^{2}+\mathrm{SMTE2}^{2}+\mathrm{DR}_{1}^{2}+\mathrm{DR}_{2}^{2}\right)^{1 / 2}=\mathrm{TS}_{\mathrm{sc}}-\mathrm{AVSTM}$

$$
\mathrm{AV}_{\mathrm{sc}}=11-01^{*}\left(0355^{2}+0254^{2}+22+002^{2}\right)^{1 / 2}=11 \quad 0224=09
$$

$\operatorname{AVSTM}=0224 \mathrm{scfm}$ 


\section{Seismic transmitter to SCIC}

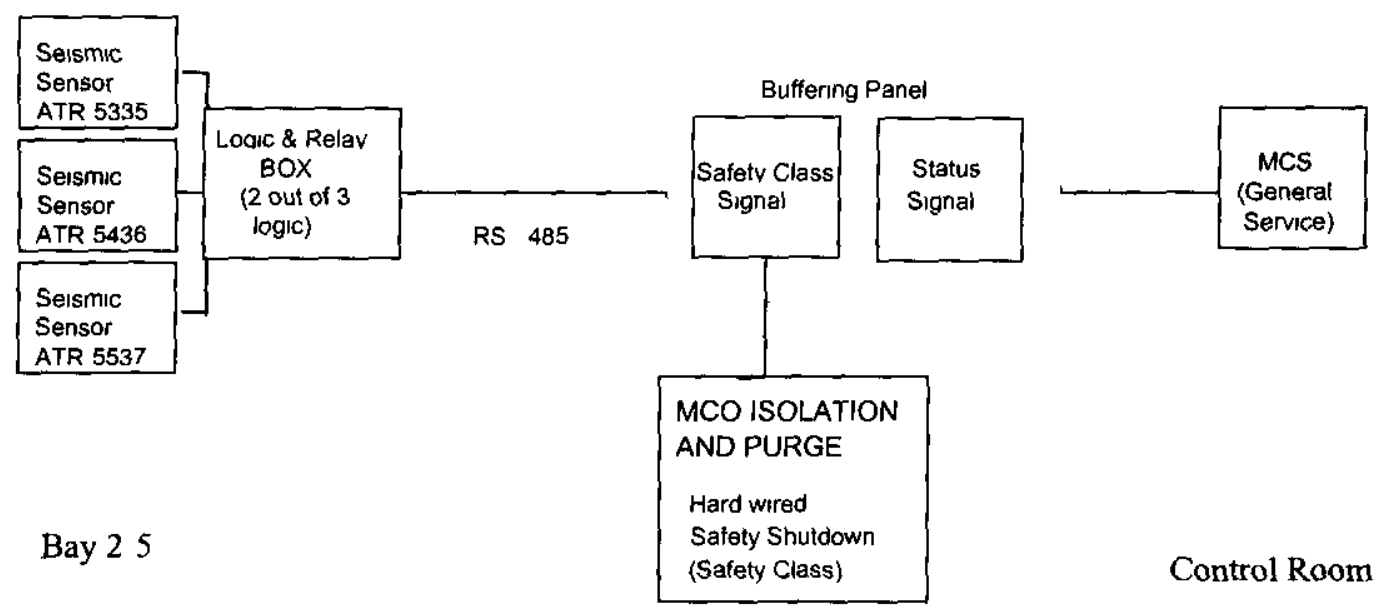

Figure 61 Seismic Sensor Simplified Block Diagram

\section{Seismic Set Point Identufication}

The seismic montoring trip design was based on the requirements

1) To reduce the seismic qualification necessary for all instruments used by the SCIC for trip functions and

2) To allow the Mode Switch to be located in a non-sersmically-qualified location (CVDF control room)

A $005 \mathrm{~g}$ value parameter limit is being used to assure a seismic trip well in advance of a seismic event of significant magnitude to break the VPS piping that is not part of the qualified primary MCO boundary This feature allowed all the piping outside the isolation valves to be general service The $005 \mathrm{~g}$ level is at a point where most people would feel the effect but no damage would occur This level is 20\% of the Design Basis Event (DBE) level of $026 \mathrm{~g}$

\section{Function}

Inputs from SCIC system seismic monitors provide indication of a safety-class seismic event Seismic recorders are used to detect, record, and trip on a seismic event exceeding the setpoint (parameter limit $005 \mathrm{~g}$, triaxial, any direction) Auctioneering logic is used to preclude fault trips by locating each of three sensors in process bays 23 , and 4 and by using two out of three (2/3) logic to initiate a Tempered Water (TW) trip and an MCO isolation and purge (ISO \& PURGE) trip The seismic recorder and trip components must initiate a signal to the SCIC system upon ground motion acceleration above the setpoint in order to shutdown the CVD system unt 1 equipment is verified operational However, the seismic trips are independent of other potions of the SCIC safety class logic The seismic trip directly controls the final output relay, which is either 
closed to allow power to the MCO 1solation valves TW heater, and PWC pumps or opened on a trip or loss of power

The PWC Seismic Trip protection is not redundant unlıke all other SCIC portions Instead Train A of the Seismic trip ctrcult feeds the starter for one of the PWC pumps (PWC-P-4035) and Train B feeds the starter for the other PWC Pump (PWC-P-4036) This protection is classified Safety Significant A combination of seismic induced pipe failures while retaining power to the pumps and a loss of power to the HVAC fans is considered unlikely

Once a seismic trip is activated, all other logic is circumvented and no longer has a safetyclass function The control room functions, both the mode switch and annunciation, may not be avallable since the control room is not seismically-qualıfied There are no control room functions required during or after a seismic event The switches and transmitters used as inputs to the SCIC are also not seismically-qualıfied for operability since none are needed for post-accident indication or control and the seismic monitoring portion of the SCIC places the process system in a safeshutdown mode

\section{Plant Operatıng Conditions}

There are three seismic sensors/recorder one each in Bay 2 Bay 3 and Bay 4 The two seismic logic and relay panels are located in Bay 1 to provide easy access for Operations and Maintenance To reduce spunous trips, the three sensors are auctioneered $(2 / 3 \log 1 \mathrm{c})$ with self resettıng trips from each sensor Reset of a single sensor trip occurs in approximately 30 seconds from the time of the last seismic level above $05 \mathrm{~g}$ If at least two of the three sensors alarm, a seismic trip occurs A seismic trip de energizes the TW heater initiates an automatic ISO \& PURGE of the MCO and de-energizes the PWC circulating pumps The Seismic trips are hardwired and always remain active

SEISMIC TRIP status indicators are provided in the respective bays These indicators are normally illuminated green when there is no trip Upon a trip condition the indicator extınguishes and stays extinguished until the condition has been reset, as described above Lamp tests are provided Reset after a seismic 2/3 trip requires a manual reset in Bay 1 for both Trains $A$ and $B$

All seismic equipment receives $120 \mathrm{VAC}$ from a non-safety source The seismic sensor/recorders include battery backup power so seismic monitoring can contınue during a power outage to record seismic activity durng/after an earthquake for analysis of the potential impacts of the earthquake e seismic monitoring sensor alarms (Units 1, 2 and 3, seismic 2/3 trips, and error alarm (Channels A and B)) are provided to the non safety MCS The seismic monitoring system components and wirıng are fail-safe, whereas fallures result in both an alarm and a trip signal (1 e , relays normally energized contacts closed)

\section{Design-basis event conditions}

1 The seismic portion of the SCIC is always active and will initiate a trip during all modes if 2 out of 3 of the seismic recorders detect a seismic event that exceeds $005 \mathrm{~g}$, triaxial, any direction 
Terra Technologies Seismic Recorders

\title{
63 Selsmic Transmitter (ATR-5335, ATR-5436, ATR-5537)
}

The sensor chosen to monitor seismic activity is a Terra Technologies SSA Series 20 Servo Accelerometer with the GSR Strong Motion recorder

\section{Servo Accelerometer}

\section{Instrument Data}

Name SSA Series 20 Servo Accelerometer

Manufacturer Terra Technologies Corp

Model Number SSA 320 (triaxial configuration)

Type Servo Accelerometer

6312 Reference Specificatıons (Specification 12/95 - Terra Technology Corp )

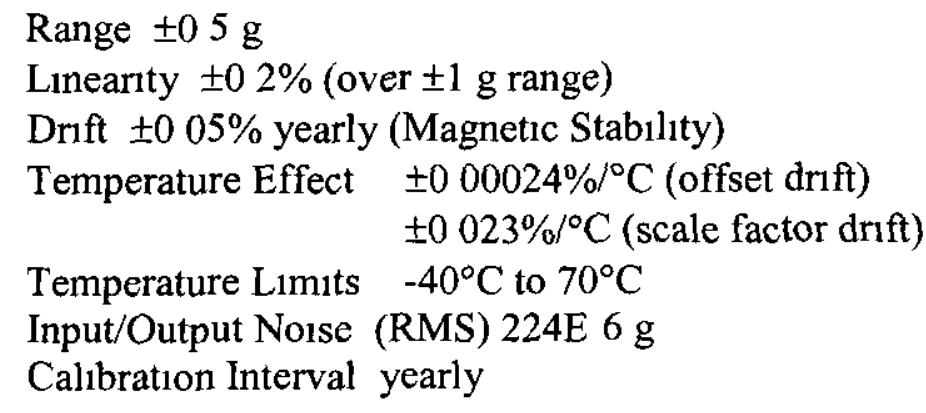

\section{Strong Motion Recorder}

6321 Instrument Data

Name Strong Motion recorder

Manufacturer Terra Technologies Corp

Model Number GSR

Type 12 bit

\section{Reference Specifications}

\author{
Range $\pm 05 \mathrm{~g}$ \\ Digitization Error $\pm 0025 \%$ FS (12 bit) \\ Temperature Effect $\quad \pm 0002 \% /{ }^{\circ} \mathrm{C}$ (temperature drift) \\ Temperature Limits $-10^{\circ} \mathrm{C}$ to $60^{\circ} \mathrm{C}$ \\ Calıbration Interval yearly
}


Terra Technologies Seismic Recorders

\section{Project Specifications}

The project safety specifications for the seismic sensor include the following

Safety Classification SC

Performance Category PC 3

Environmental Qualification Condition B

NPH Design Requirements Seismic Condition C Maintain critical function before, during and after Seismic event

Required Safety Functions Seismic tmp for H2 Explosion prevention

Runaway Reaction Prevention

634 Uncertainty Terms that Affect Set Point Determination

A Assumptions

1) Calibrations performed at ambient $72 \pm 8^{\circ} \mathrm{F}$ or $64-80^{\circ} \mathrm{F}$

11) $\mathrm{Min} / \max$ temperature range $1 \mathrm{~s} 40-115^{\circ} \mathrm{F}$

111 ) Full Scale (FS) = Range

B Sensor Uncertainty

1) Drift (DR)

$\mathrm{DR} 1= \pm 005 \%$

$\mathrm{DR}= \pm 005 \% \mathrm{FS}$

11) Relative Accuracy (RA) (linearity)

Given Repeatability $= \pm 02 \%$ Readıng $(\mathrm{Rdg})$ (Worst case Rdg $=05 \mathrm{~g}$ )

Digitization Error $= \pm 0025 \% \mathrm{FS}$

$\mathrm{RA}= \pm\left[\begin{array}{lll}0 & 2^{2}+0025^{2}\end{array}\right]^{\prime}= \pm 02016 \% \mathrm{FS}$

111 ) Humidity Effect (HE)

$\mathrm{HE}=0 \%$ Calibrated Span

Basis Housed in sealed enclosure

1v ) Temperature Effect (TE)

$$
\begin{aligned}
& \text { Given } \mathrm{TE}_{\mathrm{s} \text { od }}= \pm 000024 \% \mathrm{Rdg} /{ }^{\circ} \mathrm{C}\left(40^{\circ} \mathrm{C} \text { to }+70^{\circ} \mathrm{C}\right) \\
& \mathrm{TE}_{\mathrm{s} s \mathrm{~d}}= \pm 0023 \% \mathrm{Rdg} /{ }^{\circ} \mathrm{C} \quad\left(-40^{\circ} \mathrm{C} \text { to }+70^{\circ} \mathrm{C}\right) \\
& \mathrm{TE}_{\mathrm{g} \mathrm{sd}}= \pm 0002 \% \operatorname{Rdg} /{ }^{\circ} \mathrm{C} \quad\left(-10^{\circ} \mathrm{C} \text { to }+60^{\circ} \mathrm{C}\right)
\end{aligned}
$$

$\mathrm{T}_{\text {cal }}=72 \pm 8^{\circ} \mathrm{F}$ (calibration temperature range)

$\mathrm{T}_{\min }=40^{\circ} \mathrm{F}$

$\mathrm{T}_{\max }=115^{\circ} \mathrm{F}$

Maxımum Tdelta $=\mathrm{T}_{\max }-\mathrm{T}_{\text {cal }}($ lower $)-=115^{\circ} \mathrm{F}-64^{\circ} \mathrm{F}=51 \mathrm{delta}^{\circ} \mathrm{F}=28 \mathrm{delta}^{\circ} \mathrm{C}$ 
Terra Technologies Seismic Recorders

v) Radiation Effect (RE)

Given Dose Rate $=85 \mathrm{mR} / \mathrm{hr}$

Total Integrated Dose $=85 \mathrm{mR} / \mathrm{hr} * 43800 \mathrm{hr}=372,300 \mathrm{mR}$

No Radiation Effect Data, assumed zero because relatively small dose

Assume RE $= \pm 0 \% \mathrm{FS}$

vı ) Power Supply Effect (PSE)

Given $\mathrm{PSE}=0 \%$

vi1 ) Seismic Effect (SE)

$\mathrm{SE}=0 \%$ (Designed for seimic actıvity and digital)

v111 Sensor Measurement and Test Equipment Effect (SMTE)

Test Equipment 1 Fluke 45, DC Volts, 5 digit display

$\mathrm{RA}_{\mathrm{MTE} 1}=0025 \% \mathrm{Rdg}+2 \mathrm{digits}=0025 \%+002 \%$

$$
= \pm 0045 \% \text { URL }(T D R)= \pm 011 \% \mathrm{FS}
$$

$\mathrm{RA}_{\text {STDI }}=0 \% \mathrm{FS}$ (4 1 accuracy ratio)

$\mathrm{RD} 1=0 \% \mathrm{FS}$ (digital readout)

$\mathrm{SA} 1=(0025) * 10=025 \% \mathrm{URL}=025 \%(\mathrm{TDR})=0625 \% \mathrm{FS}$

$\mathrm{MTE} 1=\left(\mathrm{RA}_{\mathrm{MTE} 1}{ }^{2}+\mathrm{RA}_{\mathrm{STD} 1}{ }^{2}+\mathrm{RD}^{2}+\mathrm{SA} 1^{2}\right)^{1 / 2}$

$\mathrm{MTE} 1=\left(011^{2}+0^{2}+0^{2}+0625^{2}\right)^{\prime \prime}=0635 \% \mathrm{FS}$

SMTE $=$ MTE $1=0635 \% \mathrm{FS}$

1x ) Total Sensor Uncertainties

$$
\begin{aligned}
& \mathrm{e}^{+}= {\left[\mathrm{RA}^{2}+\mathrm{DR}^{2}+\mathrm{TE}^{2}+\mathrm{SP}^{2}+\mathrm{HE}^{2}+\mathrm{RE}^{2}+\mathrm{PSE}^{2}+\mathrm{SE}^{2}+\mathrm{SMTE}^{2}\right]^{\prime}+\mathrm{B} } \\
& \mathrm{e}=-\left[\mathrm{RA}^{2}+\mathrm{DR}^{2}+\mathrm{TE}^{2}+\mathrm{SP}^{2}+\mathrm{HE}^{2}+\mathrm{RE}^{2}+\mathrm{PSE}^{2}+\mathrm{SE}^{2}+\mathrm{SMTE}^{2}\right]^{\prime}+\mathrm{B} \\
& \mathrm{e}= {\left[02016^{2}+005^{2}+\left(000024^{2}+0023^{2}+0002^{2}\right)^{*} 28^{2}+0+0+0+0+0+\right.} \\
&\left.0635^{2}\right]^{\prime}+224 \mathrm{E}-6 \mathrm{~g} \\
& \mathrm{e}= \pm 09297 \% \mathrm{FS}+224 \mathrm{E} 6 \mathrm{~g}= \pm(09297 / 100 * 10+224 \mathrm{e}-6) \mathrm{g} \\
& \mathrm{e}_{\text {sens }}= \pm 000952 \mathrm{~g}
\end{aligned}
$$

\section{Set Point Determmation}

A Trip Setpoint

The Analytical Limit (AL) is the maximum seismic activity allowable before the trip operates

The Trip Setpoint (TS) TS = AL $-(\mathrm{CU}+$ margın $)$

Given $A L=007$ (buffer of 001 )

Parameter Limit $=$ PL $=0$ 07-0 01 $=006$

1) $\mathrm{TS}=\mathrm{PL}-(\mathrm{CU}+$ margin $)$

$\mathrm{TS}_{\mathrm{sc}}=006-(000952)=005 \mathrm{~g}$ 
B Allowable Value

The allowable value (AV) provides a threshold value that can be used to assess the instruments expected performance when tested The allowance between the trip setpoint and the AV includes Drift instrument calibration uncertainties, and any additional instrument uncertainties durnng normal operation that are measured during testıng The purpose for determınıng an AV is to provide a threshold value that can be used to assess the instrument s performance when tested The Allowable Value setpoint trip margin (AVSTM)

111) $\quad \mathrm{AV}_{\mathrm{sc}}=\mathrm{TS}_{\mathrm{sc}}+\left(\mathrm{SMTE}^{2}+\mathrm{DR}^{2}\right)^{1 / 2}=\mathrm{TS}_{\mathrm{sc}}+\mathrm{AVSTM}$

$A V_{s c}=005 \mathrm{~g}+\left(0635^{2}+005^{2}\right)^{1 / 2} \% \mathrm{FS}=005+000637=00564 \mathrm{~g}$

AVSTM $=00064 \mathrm{~g}$ 
Terra Technologies Seismic Recorders

\section{Differential Pressure Switch}

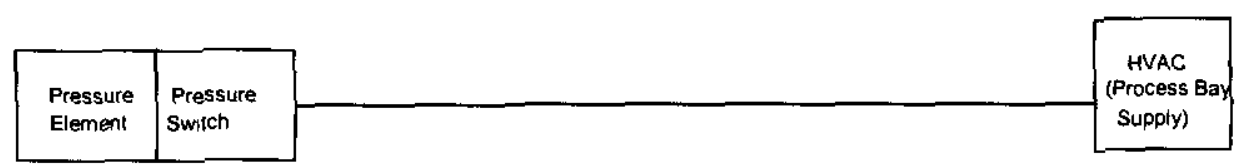

Helium

Figure 71 Dwyer Differential Pressure Switch Simplified Block Diagram

\section{Pressure Transmitter (PDIS-8022,8042,8043)}

The sensor chosen to monitor gauge pressure for the HVAC System differential pressure analytical himit is a Differential Pressure Instrument from Dwyer

\section{Instrument Data}

Name Differential Pressure Switch

Manufacturer Dwyer Instrument Inc

Model Number Series 3000MR

Type Differential Pressure Switch/Gage

712 Reference Specifications (www dwyer-1nst com/pressure/98 18p html 1998)

Range 0 to 5 in $\mathrm{H}_{2} \mathrm{O}$

Resolution 010

Accuracy $\pm 2 \%$ FS

Switch Repeatability $\pm 1 \%$

Switch Deadband $<1 \%$ FS

Calibration Interval 1 year between calibrations

Operating Temp $20^{\circ} \mathrm{F}-120^{\circ} \mathrm{F}$

\section{Project Specifications}

Safety Classification Safety Significant (SS)

Performance Category PC-2

Environmental Qualification Environmental Conditıon A

NPH Design Requirements none

Required Safety Functions Confinement 
Terra Technologies Seismic Recorders

\section{Calıbration}

A Assumptions

1) Calıbrations performed at ambient $72 \pm 8^{\circ} \mathrm{F}$ or $64-80^{\circ} \mathrm{F}$

11) $\mathrm{Min} / \max$ temperature range is $40-115^{\circ} \mathrm{F}$

111 ) Calibration Interval $=1$ year

B Calculation Verification

1) Range

$\mathrm{URL}=50$ in $\mathrm{H}_{2} \mathrm{O}$

Zero $=0$ in $\mathrm{H}_{2} \mathrm{O}$

Span $=5 \quad 0$ in $\mathrm{H}_{2} \mathrm{O}$

$\mathrm{CS}=50$ in $\mathrm{H}_{2} \mathrm{O}$

11 ) Turn Down Ratio

$$
\mathrm{TDR}=\mathrm{URL} / \mathrm{CS}=50 / 50=10
$$

111 ) Drift (Assume) $\pm 02 \%$

$$
\mathrm{DR}= \pm 02 \% *(\mathrm{URL} / \mathrm{CS})= \pm 020 \% \mathrm{CS}
$$

1v) Relative Accuracy (RA)

Assume RA $= \pm\left(\text { Resolution }^{2}+\text { Accuracy }^{2}+\text { Repeat }^{2}+\text { Deadband }^{2}\right)^{1 / 2} *$ TDR

$$
\mathrm{RA}= \pm\left(01^{2}+20^{2}+10^{2}+10^{2}\right)^{\prime}= \pm 245
$$

v) Static Pressure (SP)

$$
\mathrm{SP}=0 \% \mathrm{CS}
$$

vi Humidity Effect (HE)

$\mathrm{HE}=0 \%$ Calibrated Span

v11 ) Temperature Effect (TE)

Given $\mathrm{TE}= \pm 10 \%$ per $50^{\circ} \mathrm{F}$ ambient temperature change (Assume)

$\mathrm{T}_{\text {cal }}=72 \pm 8^{\circ} \mathrm{F}$ (calibration temperature range)

$\mathrm{T}_{\min }=40^{\circ} \mathrm{F}$

$\mathrm{T}_{\max }=115^{\circ} \mathrm{F}$

Maximum Tdelta $=\mathrm{T}_{\text {max }}-\mathrm{T}_{\text {cal }}($ lowe $)=115^{\circ} \mathrm{F}-64^{\circ} \mathrm{F}=51$ delta $^{\circ} \mathrm{F}$

$\mathrm{TE}= \pm 10 \%$ URL(TDR)

v111 ) Radiation Effect (RE)

Given $\pm 80 \%$ URL at $22 \times 10^{7}$ rads TID

Given Expected Dose Rate $=85 \mathrm{mR} / \mathrm{hr}$

$\mathrm{TID}=85 \mathrm{mR} / \mathrm{hr} * 43800 \mathrm{hr}=372,300 \mathrm{mR}=372 \times 10^{2} \mathrm{rads}$ TID

Approximate as zero because relatıvely small dose

$\mathrm{RE}= \pm 0 \% \mathrm{CS}$ 
Terra Technologies Seısmic Recorders

SNF-4451 Rev 1 Page 44 of 50

1x ) Power Supply Effect (PSE)

$\mathrm{PSE}=0 \% \mathrm{CS}$

x ) Seismic Effect (SE)

No credit for this device is taken for a safety system shutdown during or after a seismic event

$\mathrm{SE}=0 \%$

xı ) Sensor Measurement and Test Equipment Effect (SMTE)

Test Equipment 1 -Fluke 45 DC voltage mode with a 5 digit display

$\mathrm{RA}_{\mathrm{MTE}}=0025 \% \mathrm{Rdg}+2 \mathrm{digits}=0025+002$ $= \pm 0045 \% \mathrm{CS}$

$\mathrm{RA}_{\mathrm{STD} 1}=0 \% \mathrm{CS}$

$\mathrm{RD} 1=0 \% \mathrm{CS}$

$\mathrm{SA} 1=(0025 / 10) * 100=025 \% \mathrm{CS}$

$\mathrm{MTE} 1=\left(\mathrm{RA}_{\mathrm{MTEl}}{ }^{2}+\mathrm{RA}_{\mathrm{STDI}}{ }^{2}+\mathrm{RDI}^{2}+\mathrm{SAl}^{2}\right)^{1 / 2}$

MTE1 $=\left(0045^{2}+0^{2}+0^{2}+025^{2}\right)^{\prime}=0254$

Test Equipment2, Heise PTE-1 with HQS 2 sensor $\left( \pm 050\right.$ in $\left.\mathrm{H}_{2} \mathrm{O}\right)$

Accuracy $= \pm 01 \%$ span

Repeatability $= \pm 005 \%$ span

Temperature Effect $= \pm 0004 \%$ span per ${ }^{\circ} \mathrm{F}$

$\mathrm{CS}_{\text {test }}= \pm 05 \mathrm{nnH}_{2} \mathrm{O}$

$\mathrm{CS}_{\text {Inst }}= \pm 05 \mathrm{nH}_{2} \mathrm{O}$

$\mathrm{RA}_{\mathrm{MTE2} 2}= \pm\left(\mathrm{Acc}^{2}+\text { Repeat }^{2}+\mathrm{TempEffect}^{2}\right)^{1 / 2} * \mathrm{CS}_{\text {test }} / \mathrm{CS}_{\text {Inst }}= \pm 011 \% \mathrm{CS}$

$\mathrm{RA}_{\mathrm{STD} 2}= \pm 002 \% \mathrm{CS}$

$\mathrm{RD} 2=0 \% \mathrm{CS}$ (digital readout)

$\mathrm{SA} 2=0 \% \mathrm{CS}$

$\mathrm{MTE} 2=\left(\mathrm{RA}_{\mathrm{MTE} 2}{ }^{2}+\mathrm{RA}_{\mathrm{STD2}}{ }^{2}+\mathrm{RD} 2^{2}+\mathrm{SA} 2^{2}\right)^{1 / 2}$

MTE2 $=\left(026^{2}+002^{2}+0^{2}+0^{2}\right)^{\prime}=026$

SMTE $=\left[\text { MTE } 1^{2}+\text { MTE2 }^{2}\right]^{1 / 2}=\left[0254^{2}+011^{2}\right]^{1 / 2}=0277$

xi1 ) Total Sensor Uncertaint1es

$$
\begin{aligned}
& \begin{aligned}
\mathrm{e}^{+} & =\left[\mathrm{RA}^{2}+\mathrm{DR}^{2}+\mathrm{SP}^{2}+\mathrm{TE}^{2}+\mathrm{HE}^{2}+\mathrm{RE}^{2}+\mathrm{PSE}^{2}+\mathrm{SE}^{2}+\mathrm{SMTE}^{2}\right]^{\prime}+\mathrm{B} \\
& =\left[245^{2}+02^{2}+0+10^{2}+0+0+0^{2}+0+0278^{2}\right]^{1 / 2}+0=267 \% \mathrm{CS}
\end{aligned} \\
& \begin{aligned}
\mathrm{e} & =-\left[\mathrm{RA}^{2}+\mathrm{DR}^{2}+\mathrm{SP}^{2}+\mathrm{TE}^{2}+\mathrm{HE}^{2}+\mathrm{RE}^{2}+\mathrm{PSE}^{2}+\mathrm{SE}^{2}+\mathrm{SMTE}^{2}\right]^{\prime}+\mathrm{B} \\
& =\left[245^{2}+02^{2}+0+10^{2}+0+0+0^{2}+0+0278^{2}\right]^{1 / 2}+0=-267 \% \mathrm{CS}
\end{aligned} \\
& \mathrm{e}_{\text {sens }}= \pm 267 \% \mathrm{CS} \\
& \mathrm{e}_{\text {sens }}= \pm(00267)^{*} 05 \text { in } \mathrm{H}_{2} \mathrm{O}= \pm 0013 \text { in } \mathrm{H}_{2} \mathrm{O}
\end{aligned}
$$


Reotemp Pressure Indicator - Local Pressure

8 He Bottle Pressure Indicator

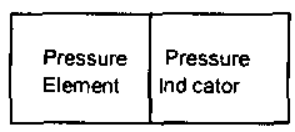

He Bottle

PRESSURE

Figure 81 Reotemp Gauge Pressure Simplified Block Diagram

\section{Gauge Pressure Indicator (PI-5*02, PT-5*21, PT-5*41, PT-5*61)}

The sensor chosen to monitor pressure for the 2500 psig SC helium bottle pressure analytical limit is Gauge Pressure Indicators from Reotemp Instrument Corp

811 Instrument Data

Name Pressure Gauge

Manufacturer Reotemp Instrument Corp

Model Number PR 25-S 1-A-4-P32-D

Type Local Pressure Gauge

812 Reference Specifications

Range $0-3000$ psig

Resolution N/A

Accuracy $\pm 16 \%$ FS

Calibration Interval 1 year between calibrations

\section{Project Specifications}

Safety Classification Safety Class (SC)

Performance Category PC-3

Environmental Qualification Environmental Condition B

NPH Design Requirements Seismic Condition A

Required Safety Functions Pressure boundary integrity Post Accident Monitoring

814 Calibration

A Assumptions

1) Calibrations performed at ambient $72 \pm 8^{\circ} \mathrm{F}$ or $64-80^{\circ} \mathrm{F}$

11) $\mathrm{Min} / \max$ temperature range is $40-115^{\circ} \mathrm{F}$

111 ) Calibration Interval $=1$ year

B Calculation Verification 
Reotemp Pressure Indicator - Local Pressure

1) Range

$$
\begin{aligned}
& \text { URL }=3000 \text { psig } \\
& \text { Zero }=0 \text { psig } \\
& \text { Span }=3000 \text { psig } \\
& \text { CS }=3000 \text { psig }
\end{aligned}
$$

11 ) Turn Down Rat1o

$\mathrm{TDR}=\mathrm{URL} / \mathrm{CS}=3000 / 3000=10$

111 ) Drift

Assume $\pm 1 \%$

$\mathrm{DR}= \pm 1 \% *(\mathrm{URL} / \mathrm{CS})= \pm 1 \% \mathrm{CS}$

1v ) Relative Accuracy (RA)

Given $\mathrm{RA}= \pm 16 \% \mathrm{CS}$

v) Static Pressure (SP)

$\mathrm{SP}=0 \% \mathrm{CS}$

v1 ) Humidity Effect (HE)

$\mathrm{HE}=0 \%$ Calibrated Span

Basis $0-100 \%$ relative humidity (Housed in sealed enclosure)

vi1 ) Temperature Effect (TE)

Given Operatıng Temperature $-30^{\circ} \mathrm{F}$ to $400^{\circ} \mathrm{F}$

Assume $\mathrm{TE}= \pm 0 \% \mathrm{URL}$

v111 ) Radiation Effect (RE)

Given $\pm 80 \%$ URL at $22 \times 10^{7}$ rads TID

Given Expected Dose Rate $=85 \mathrm{mR} / \mathrm{hr}$

$\mathrm{TID}=85 \mathrm{mR} / \mathrm{hr} * 43800 \mathrm{hr}=372,300 \mathrm{mR}=372 \times 10^{2} \mathrm{rads}$ TID

Approximate as zero because relatively small dose

$\mathrm{RE}= \pm 0 \% \mathrm{CS}$

1x ) Power Supply Effect (PSE)

Given $>0005 \%$ of output span/volt

PSE $>0001 \%$ CS

x ) Seismic Effect (SE)

No credit for this device is taken for a safety system shutdown during or after a seismic event

$\mathrm{SE}=0 \%$ 
Reotemp Pressure Indicator - Local Pressure

xi ) Sensor Measurement and Test Equipment Effect (SMTE)

Test Equipment1 Heise PTE-1 with HQS-2 sensor (0-3000psig)

Accuracy $= \pm 01 \%$ span

Repeatability $= \pm 002 \%$ span

Temperature Effect $= \pm 0004 \%$ span per ${ }^{\circ} \mathrm{F}$

$\mathrm{CS}_{\text {test }}=3000 \mathrm{psig}$

$\mathrm{CS}_{\text {inst }}=3000 \mathrm{psig}$

$\mathrm{RA}_{\mathrm{MTE} 1}= \pm\left(\mathrm{Acc}^{2}+\text { Repeat }^{2}+\mathrm{TempEffect}^{2}\right)^{1 / 2} * \mathrm{CS}_{\mathrm{test}} / \mathrm{CS}_{\text {Inst }}= \pm 022 \% \mathrm{CS}$

$\mathrm{RA}_{\mathrm{STD1}}= \pm 002 \% \mathrm{CS}$

$\mathrm{RD} 1=0 \% \mathrm{CS}$ (digital readout)

$\mathrm{SA} 1=0 \% \mathrm{CS}$

$\mathrm{MTE} 1=\left(\mathrm{RA}_{\mathrm{MTEl}}{ }^{2}+\mathrm{RA}_{\mathrm{STD1}}{ }^{2}+\mathrm{RD}^{2}+\mathrm{SAl}^{2}\right)^{1 / 2}$

MTE1 $=\left(022^{2}+002^{2}+0^{2}+0^{2}\right)^{\prime}=022$

$\mathrm{SMTE}=022$

xi1 ) Total Sensor Uncertaint1es

$$
\begin{aligned}
& \mathrm{e}^{+}=\left[\mathrm{RA}^{2}+\mathrm{DR}^{2}+\mathrm{SP}^{2}+\mathrm{TE}^{2}+\mathrm{HE}^{2}+\mathrm{RE}^{2}+\mathrm{PSE}^{2}+\mathrm{SE}^{2}+\mathrm{SMTE}^{2}\right]^{\prime}+\mathrm{B} \\
& =\left[16^{2}+10^{2}+0+0^{2}+0+0+0^{2}+0+022^{2}\right]^{1 / 2}+0=19 \% \mathrm{CS} \\
& \mathrm{e}=-\left[\mathrm{RA}^{2}+\mathrm{DR}^{2}+\mathrm{SP}^{2}+\mathrm{TE}^{2}+\mathrm{HE}^{2}+\mathrm{RE}^{2}+\mathrm{PSE}^{2}+\mathrm{SE}^{2}+\mathrm{SMTE}^{2}\right]^{\prime}+\mathrm{B} \\
& =-\left[16^{2}+10^{2}+0+0^{2}+0+0+0^{2}+0+022^{2}\right]^{1 / 2}+0=-19 \% \mathrm{CS} \\
& \mathrm{e}_{\mathrm{sens}}= \pm 19 \% \mathrm{CS} \\
& \mathrm{e}_{\mathrm{sens}}= \pm(0019)^{*} 3000 \mathrm{psig}= \pm 56 \mathrm{psig}
\end{aligned}
$$


W E Anderson (Dwyer) Flow Switch

9 Flow Switch

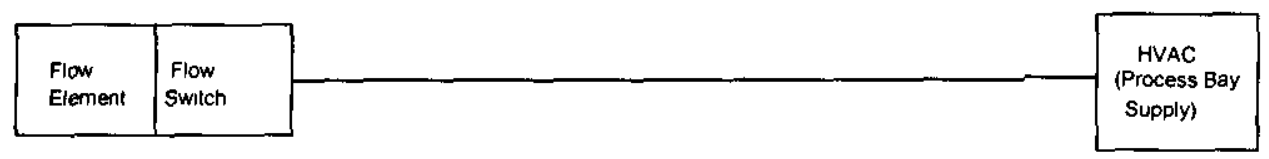

Helium

Figure 91 W E Anderson (Dwyer) Vane Operated Flow Switch Simplified Block Diagram

\section{Flow Switch $($ FS-8*52)}

The sensor chosen to monitor flow and operate flow switches for the HVAC System is a Vane Operated Flow Switch from W E Anderson (Dwyer)

911 Instrument Data

Name Series V4 Flotect Vane Operated Flow Switch

Manufacturer Dwyer Instrument Inc

Model Number V4-SS

Type Flow Switch

912 Reference Specifications (www dwyer inst com/flow/98-22f html 1998)

Range N/A

Accuracy $\pm 10 \%$ FS

Calıbration Interval 1 year between calibrations

Operating Temp up to $275^{\circ} \mathrm{F}$

913 Calıbration

A Assumptions

1) Calibrations performed at ambient $72 \pm 8^{\circ} \mathrm{F}$ or $64-80^{\circ} \mathrm{F}$

11 ) $\mathrm{Min} / \max$ temperature range $1 \mathrm{~s} 40-115^{\circ} \mathrm{F}$

111 ) Calibration Interval $=1$ year

B Calculation Verification

1) Range $=N / A$

$\mathrm{CS}=\mathrm{N} / \mathrm{A}$

11) Turn Down Ratio

$\mathrm{TDR}=\mathrm{URL} / \mathrm{CS}=10$

111 ) Drift

$\mathrm{DR}= \pm 0 \%$ 
W E Anderson (Dwyer) Flow Switch

Iv ) Relatıve Accuracy (RA)

$\mathrm{RA}= \pm 10 \%$

v) Static Pressure (SP)

$\mathrm{SP}=0 \% \mathrm{CS}$

vi ) Humidity Effect (HE)

$\mathrm{HE}=0 \%$ Calıbrated Span

vil ) Temperature Effect (TE)

Assume $\mathrm{TE}= \pm 0 \%$ per ${ }^{\circ} \mathrm{F}$ over expected range

v111 ) Radiation Effect (RE)

Given $\pm 80 \%$ URL at $22 \times 10^{7}$ rads TID

Given Expected Dose Rate $=85 \mathrm{mR} / \mathrm{hr}$

$\mathrm{TID}=85 \mathrm{mR} / \mathrm{hr}^{*} 43800 \mathrm{hr}=372300 \mathrm{mR}=372 \times 10^{2}$ rads TID

Approximate as zero because relatively small dose

$\mathrm{RE}= \pm 0 \% \mathrm{CS}$

Ix ) Power Supply Effect (PSE)

PSE $=0 \%$ CS

x ) Seismic Effect (SE)

No credit for this device is taken for a safety system shutdown during or after a seismic event

$\mathrm{SE}=0 \%$

x1 ) Sensor Measurement and Test Equipment Effect (SMTE)

Test Equipment 1 -Calıbration laboratory instrumentation

Accuracy $=1 \% \operatorname{Rdg}$ (NIST Traceable)

Parameter Lımit $=1000 \mathrm{scfm}$

Calibrated Span $=1200 \mathrm{scfm}$

$\mathrm{SMTE}= \pm 1 \% \mathrm{CS}$ (assume)

x11 ) Total Sensor Uncertaintıes

$$
\begin{aligned}
& \begin{aligned}
\mathrm{e}^{+} & =\left[\mathrm{RA}^{2}+\mathrm{DR}^{2}+\mathrm{SP}^{2}+\mathrm{TE}^{2}+\mathrm{HE}^{2}+\mathrm{RE}^{2}+\mathrm{PSE}^{2}+\mathrm{SE}^{2}+\mathrm{SMTE}^{2}\right]^{\prime}+\mathrm{B} \\
& =\left[10^{2}+0^{2}+0+0^{2}+0+0+0^{2}+0+1^{2}\right]^{1 / 2}+0=10 \% \mathrm{CS}
\end{aligned} \\
& \begin{aligned}
\mathrm{e} & =-\left[\mathrm{RA}^{2}+\mathrm{DR}^{2}+\mathrm{SP}^{2}+\mathrm{TE}^{2}+\mathrm{HE}^{2}+\mathrm{RE}^{2}+\mathrm{PSE}^{2}+\mathrm{SE}^{2}+\mathrm{SMTE}^{2}\right]^{\prime}+\mathrm{B} \\
& =-\left[10^{2}+0^{2}+0+0^{2}+0+0+0^{2}+0+1^{2}\right]^{1 / 2}+0=10 \% \mathrm{CS}
\end{aligned} \\
& \mathrm{e}_{\text {sens }}= \pm 10 \% \mathrm{CS} \\
& \mathrm{e}_{\text {sens }}= \pm(010)^{*} 1200 \mathrm{scfm}= \pm 120 \mathrm{scfm}
\end{aligned}
$$


W E Anderson (Dwyer) Flow Switch

\section{References}

\section{Vender Information}

1 Bulletın 400-10/96 1996 - MKS Instruments, Inc )

2 letter from R Traverso MKS Applications Engıneer 1/26/98

3 1960501R 5/96 - Framatome Technologies

4 PDS 4302 Apr11 1992 Rosemount Nuclear

5 SW/PI-62 4/3/95 - Ashcroft

6 SW-12-Ashcroft

7 letter 11/10/97 from H Sittınger App Engineer FCI

8 Qualıfication Report \#708349, 8/20/84 - FCI Fluid Components, Inc )

9 Specification 12/95 - Terra Technology Corp

10 www dwyer-1nst com/pressure/98-18p html 1998

11 www dwyer-ınst com/flow/98-22f html, 1998

\section{HANFORD}

1 SNF-3091 1999, Cold Vacuum Drying Safety Class Instrumentatıon and Control System Design Description Rev 1, Fluor Hanford, Inc, Richland Washington

2 HNF-1851, Cold Vacuum Drying Residual Free Water Test Description Fluor Hanford Inc, Richland, Washıngton

3 HNF-SD SNF-DRD 002 Rev 4, Cold Vacuum Drying Facllty Design Requirements Fluor Hanford Inc, Richland Washington 